\title{
Article \\ Emergence of Unique SARS-CoV-2 ORF10 Variants and Their Impact on Protein Structure and Function
}

\author{
Sk. Sarif Hassan ${ }^{1} * \mathbb{D}$, Kenneth Lundstrom ${ }^{2}$, Ángel Serrano-Aroca ${ }^{3}$, Parise Adadi ${ }^{4}$, Alaa A. A. Aljabali ${ }^{5}$, Elrashdy M. \\ Redwan ${ }^{6,7}$, Amos Lal ${ }^{8}$, Ramesh Kandimalla9 , Tarek Mohamed Abd El-Aziz ${ }^{10,11}$, Pabitra Pal Choudhury ${ }^{12}$, Gajendra \\ Kumar Azad $^{13}$, Samendra Sherchan ${ }^{14}$, Murtaza Tambuwala ${ }^{15}$, Gaurav Chauhan ${ }^{16}$, Kazuo Takayama ${ }^{17}$, Debmalya \\ Barh $^{18,19}$, Giorgio Palù ${ }^{20}$, Pallab Basu ${ }^{21}$, Vladimir Uversky ${ }^{22, * \mathbb{D}}$
}

1 Department of Mathematics, Pingla Thana Mahavidyalaya, Maligram, Paschim Medinipur 721140, West Bengal, India; sksarifhassan@pinglacollege.ac.in

2 PanTherapeutics, Rte de Lavaux 49, CH1095 Lutry, Switzerland; lundstromkenneth@gmail.com

3 Biomaterials and Bioengineering Lab, Centro de Investigación Traslacional San Alberto Magno, Universidad Católica de Valencia San Vicente Mártir, c/Guillem de Castro, 94, 46001 Valencia, Valencia, Spain; angel.serrano@ucv.es

4 Department of Food Science, University of Otago, Dunedin 9054, New Zealand; pariseadadi@gmail.com

5 Department of Pharmaceutics and Pharmaceutical Technology, Faculty of Pharmacy, Yarmouk University, Irbid 566, Jordon; alaaj@yu.edu.jo

6 Biological Science Department, Faculty of Science, King Abdulaziz University, Jeddah, Saudi Arabia; lradwan@kau.edu.sa

7 Therapeutic and Protective Proteins Laboratory, Protein Research Department, Genetic Engineering and Biotechnology Research Institute, City of Scientific Research and Technological Applications, New Borg EL-Arab, 21934, Alexandria, Egypt

8 Department of Medicine, Division of Pulmonary and Critical Care Medicine, Mayo Clinic, Rochester, Minnesota, USA; manavamos@gmail.com

9 Applied Biology, CSIR-Indian Institute of Chemical Technology, Uppal Road, Tarnaka, Hyderabad-500007, Telangana State, India; ramesh.kandimalla@gmail.com

10 Department of Cellular and Integrative Physiology, University of Texas Health Science Center at San Antonio, 7703 Floyd Curl Dr, San Antonio, TX 78229-3900, USA; mohamedt1@uthscsa.edu

11 Zoology Department, Faculty of Science, Minia University, El-Minia 61519, Egypt

12 Applied Statistics Unit, Indian Statistical Institute, Kolkata 700108, India; pabitra@isical.ac.in

13 Department of Zoology, Patna University, Patna, Bihar, India; gkazad@patnauniversity.ac.in

14 Department of Environmental Health Sciences, Tulane University, New Orleans, LA 70112, USA; sshercha@tulane.edu

15 School of Pharmacy and Pharmaceutical Science, Ulster University, Coleraine BT52 1SA, Northern Ireland, UK; m.tambuwala@ulster.ac.uk

16 School of Engineering and Sciences, Tecnologico de Monterrey, Av. Eugenio Garza Sada 2501 Sur, 64849 Monterrey, Nuevo León, Mexico; gchauhan@tec.mx

17 Center for iPS Cell Research and Application (CiRA), Kyoto University, Kyoto 606-8507, Japan; kazuo.takayama@cira.kyoto-u.ac.jp

18 Centre for Genomics and Applied Gene Technology, Institute of Integrative Omics and Applied Biotechnology (IIOAB), Nonakuri, Purba Medinipur, WB, India; dr.barh@gmail.com

19 Departamento de Genética, Ecologia e Evolucao, Instituto de Ciências Biológicas, Universidade Federal de Minas Gerais, Belo Horizonte, Minas Gerais, Brazil

20 Department of Molecular Medicine, University of Padova, Via Gabelli 63, 35121, Padova, Italy; giorgio.palu@unipd.it

21 School of Physics, University of the Witwatersrand, Johannesburg, Braamfontein 721140, South Africa; pallabbasu@gmail.com

22 Department of Molecular Medicine, Morsani College of Medicine, University of South Florida, Tampa, FL 33612, USA; vuversky@usf.edu

* Correspondence: vuversky@usf.edu; sksarifhassan@pinglacollege.ac.in.

\begin{abstract}
The devastating impact of the ongoing coronavirus disease 2019 (COVID-19) on public health, caused by the Severe Acute Respiratory Syndrome Coronavirus 2 (SARS-CoV-2) has made fighting of the COVID-19 pandemic is a top priority in medical research and pharmaceutical development. Surveillance of SARS-CoV-2 mutations is essential for the comprehension of SARS$\mathrm{CoV}-2$ variant diversity and their impact on virulence and pathogenicity. The SARS-CoV-2 open reading frame 10 (ORF10) protein interacts with multiple human proteins CUL2, ELOB, ELOC,
\end{abstract}


MAP7D1, PPT1, RBX1, THTPA, TIMM8B, and ZYG11B expressed in the lung tissues. Mutations and co-mutations in the emerging SARS-CoV-2 ORF10 variants are expected to impact the severity of the virus and its associated consequences. In this article, We highlight 128 single mutations and 35 co-mutations in the unique SARS-CoV-2 ORF10 variants in this article. The possible predicted effects of these mutations and co-mutations on the secondary structure of ORF10 variants and host protein interactomes are presented. The findings highlight the possible effects of mutations and co-mutations on the emerging 140 ORF10 unique variants from secondary structure and intrinsic protein disorder perspectives.

Keywords: SARS-CoV-2, ORF10, Co-mutations, Intrinsic Protein Disorder, and Ubiquitin Ligase Complex

\section{Introduction}

Severe Acute Respiratory Syndrome Coronavirus 2 (SARS-CoV-2) continues the pandemic spread of coronavirus disease 2019 (COVID-19), with over 189 million people confirmed infected and at least 4.07 million deaths worldwide [1,2]. In 2021, in almost every region of the SARS-CoV-2 genome, several mutations compared to the wild SARS-CoV-2 (NC_045512) were discovered [3-5]. The SARS-CoV-2 is constantly evolving and new variants with different characteristics are emerging [6,7]. Detection and mutation surveillance of SARS-CoV-2 is of utmost priority investigate the origin and to combat the virus [8]. To date, no method can rapidly diagnose multiple viral infections and determine variants in a high-throughput manner [9]. SARS-CoV-2 is one of the largest RNA viruses with a genome of approximately $29 \mathrm{~Kb}$, which includes eleven open reading frames (ORFs) [10-13]. These ORFs ( $1 \mathrm{a}$ and $1 \mathrm{~b})$ possessed two polypeptides that are translated into sixteen non-structural proteins (NSP1-16) [14-16]. The main nonstructural proteins (NSP) include RNA-dependent RNA polymerase (RdRp or NSP12) and a $3^{\prime}-5^{\prime}$ exonuclease [17]. RNA viruses typically possess a high mutation rate ranging from $10^{-6}$ to $10^{-4}[18,19]$. Notably, SARS-CoV-2 possesses the $3^{\prime}-5^{\prime}$ exonuclease capable of correcting mistakes during replication [20]. While surveilling mutations to comprehend the genetic diversity across various SARS-CoV-2 variants, it is also important to decipher whether the increase in mutation frequency is because of the natural selection, and to determine the possible consequences for SARS-CoV-2 fitness, such as increased infectivity and pathogenicity, or due to adaptation, thereby becoming drug-resistant or possessing ability to evade the immune system [21,22]. Non-synonymous mutations of various SARS-CoV-2 proteins have been reported [23-26].

The ORF proteins are dispensable for viral growth in vitro, and might play an important roles within the environment of the infected host [27]. The SARS-CoV-2 open reading frame 10 (ORF10) protein shows no sequence similarity with other known coronavirus protein [28-30]. The SARS-CoV-2 ORF10, a putative 38-amino acid viral protein encoded in the 3 ' accessory region of the genome, is a highly ordered, hydrophobic, and thermally stable protein, which contains at least one transmembrane region $[29,31]$. The ORF10 binds to components of a Cullin-2-RING-ligase (CRL2) complex containing Cullin-2, RBX1, Elongin B, Elongin C, and ZYG11B (CRL2 $\left.{ }^{Z Y G 11 B}\right)$ [32-34]. Earlier, it has been reported that the extreme $\mathrm{N}$ terminus of ORF10 contains a methionine-glycinetyrosine motif, which would presumably aid ORF10 to be recruited into CRL2 ${ }^{Z Y G 11 B}$ ubiquitin ligase complex [33]. It was further confirmed that interaction between ORF10 and $C R L 2^{Z Y G 11 B}$ is not relevant for SARS-CoV-2 infection in vitro [30,33]. No evidence was observed whether ORF10 regulates or is regulated by $C R L 2^{Z Y G 11 B}$ [33]. On the other hand, ORF10 protein in some SARS-CoV-2 variants, resulted in non-attenuation of disease and maintained transmissibility [35]. Furthermore, ORF10 is not essential for viral infection and replication while encoding a truncated protein that is neutrally 
evolving, through positive selection $[30,36]$.

In this study, we report 128 single mutations and 35 co-mutations in the unique SARS-CoV-2 ORF10 variants. This report illuminates potential effects due to mutations and co-mutations in the emerging ORF10 variants from the secondary structure and intrinsic protein disorder perspectives.

\section{Materials and Methods}

\subsection{Data}

A total of 202968 SARS-CoV-2 ORF10 sequence data were retrieved from the National Center for Biotechnology Information (NCBI) database on June 29, 2021. Note that none of these complete ORF10 sequences contain any ambiguous characters. Among 202968 SARS-CoV-2 ORF10 sequences only 140 sequences were unique and distinct, globally. Furthermore, SARS-CoV-2 ORF10 protein sequences from the GISAID database were considered for finding co-mutations from the CoVal database.

\subsection{Methods}

2.2.1. Transmembrane topology and secondary structure prediction

Prediction of a transmembrane protein topology of a given protein is one of the classical issues in bioinformatics. The Phobius program was used to predict transmembrane topology for SARS-CoV-2 ORF10 protein variants $[37,38]$.

The secondary structure of the SARS-Cov-2 ORF10 protein was predicted using the JPred 4 webserver [39]. In addition to protein secondary structure JPred was used to predict solvent accessibility and coiled-coil regions. The following keys were used in the secondary structure prediction of ORF10 variants [39].

- Shades of red: The more red a position is, the higher the level of conservation of chemical properties of the amino acids.

- Jnetpred: Final secondary structure prediction for a query.

- Jnet_25: Jnet prediction of burial, less than $25 \%$ solvent accessibility.

- Jnet_5: Jnet prediction of burial, less than 5\% exposure.

- Jnet_0: Jnet prediction of burial, $0 \%$ exposure.

- Jnetconf: Jnet reliability of prediction accuracy ranges from 0 to 9 , bigger is better.

\subsubsection{Intrinsic disorder analysis}

All SARS-CoV-2 ORF10 variants were subjected to the per-residue disorder analysis, with PONDR-VSL2 algorithm [40]. This tool showed good performance on proteins containing both structure and disorder, and is considered as an accurate standalone disorder predictor [41-43]. Predisposition scores for the per-residual conditions are 0 to 1 , where 0 indicates residues entirely arranged, and 1 indicates residues completely disordered. Residues with disorder scores between 0.25 and 0.5 were considered as disordered, residues with disorder scores between 0.1 and 0.25 were considered moderately disordered, whereas residues with values higher than 0.5 were considered disordered.

\subsubsection{Analysis of sequence variation}

Single mutations in all the 140 unique ORF10 proteins were determined using the Virus Pathogen Resource ViPR by uploading a Fasta file of ORF10 sequences [44]. A snapshot of the ORF10 sequence variations is presented in Figure 1. 


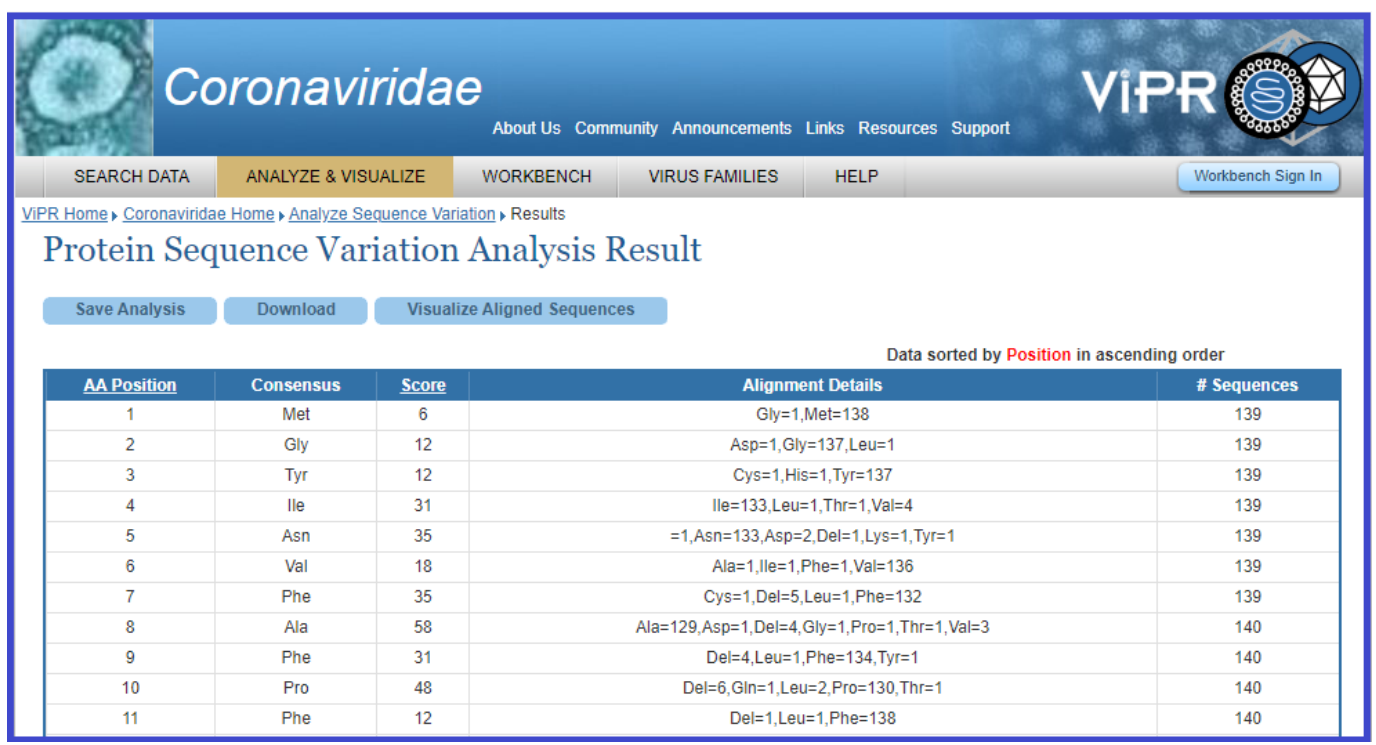

Figure 1. A snapshot of ViPR database showing the ORF10 sequence variations and consensus amino acid residues.

Furthermore, the predicted effect on pathogenicity of all the mutations was analyzed with PredictSNP and PhD-SNP $[45,46]$. In addition, co-occurrence of mutations in ORF10 proteins were also detected by the CoVal database.

A quantitative measure was defined to localize the co-occurrent mutations in an ORF10 protein in a given geo-location with regards to other geo-locations worldwide.

Localization across countries: For each co-occurrent mutation in an ORF10 protein, localization is defined as

$$
N F_{m}=\frac{N G_{m}}{T G_{m}}
$$

Where, $N G_{m}$ and $T G_{m}$ denote the number of SARS-CoV-2 genomes with these specific simultaneous mutations $(m)$ in a geo-location, and the total number of SARS-CoV-2 genomes with this mutation $(m)$ worldwide, respectively. It varies from 0 to 1 . The normalized factor 0 denotes uniformly spreading of the mutations across various geolocations, whereas 1 denotes the detection of the mutation in a single geo-location (discussed in CoVal database).

\subsubsection{Frequency distribution of amino acids and clustering}

The frequency distribution of each amino acid present in ORF10 sequence was determined using standard bioinformatics routine in Matlab [47]. For each ORF10 sequence, a twenty-dimensional frequency-vector considering the frequency of standard twenty amino acids can be obtained. The distance (Euclidean metric) between any two pairs of frequency vectors was calculated for each pair of ORF10 sequences. By having the distance matrix, a set of clusters was obtained applying the well-known K-means clustering method using the standard routine in Matlab-2021a [48,49].

\section{Results}

3.1. Continent-wise unique ORF10 variants and mutations

Continent-wise unique variations of ORF10 proteins and their mutations with predicted effects are presented in the following subsections. 


\subsubsection{ORF10 variants and transmembrane topology of SARS-CoV-2 ORF10}

Continent-wise distribution of the total 140 unique ORF10 variants are presented in Table 1. The highest frequency of unique ORF10 variants was found in North America among the total of 140 SARS-CoV-2 ORF10 variants.

Table 1: Unique mutations in the unique ORF10 variants across six continents. \# denotes "number of"

\begin{tabular}{ccccccc}
\hline & Africa & Asia & Europe & North America & Oceania & South America \\
\hline \# of total ORF10 & 1183 & 3393 & 1033 & 186572 & 10290 & 497 \\
\# of unique ORF10 & 15 & 21 & 7 & 132 & 15 & 6 \\
\% of unique ORF10 & 1.27 & 0.62 & 0.68 & 0.07 & 0.15 & 1.21 \\
\# of residue positions & 12 & 15 & 6 & 37 & 12 & 5 \\
of mutations in ORF10 & 31.6 & 39.5 & 15.8 & 97.4 & 31.6 & 13.2 \\
\hline \% of total number of residues & & & & & &
\end{tabular}

Continent-wise, a decreasing order percentage of unique ORF10 variants was Africa $>$ South America $>$ Europe $>$ Asia $>$ Oceania $>$ North America (Table 1).

In addition, the transmembrane topology and signal peptides for the wild-type SARS-CoV-2 ORF10 (YP_009725255) protein was predicted using the Phobius webserver. The associated posterior probability for the topology prediction is presented in Figure 2.

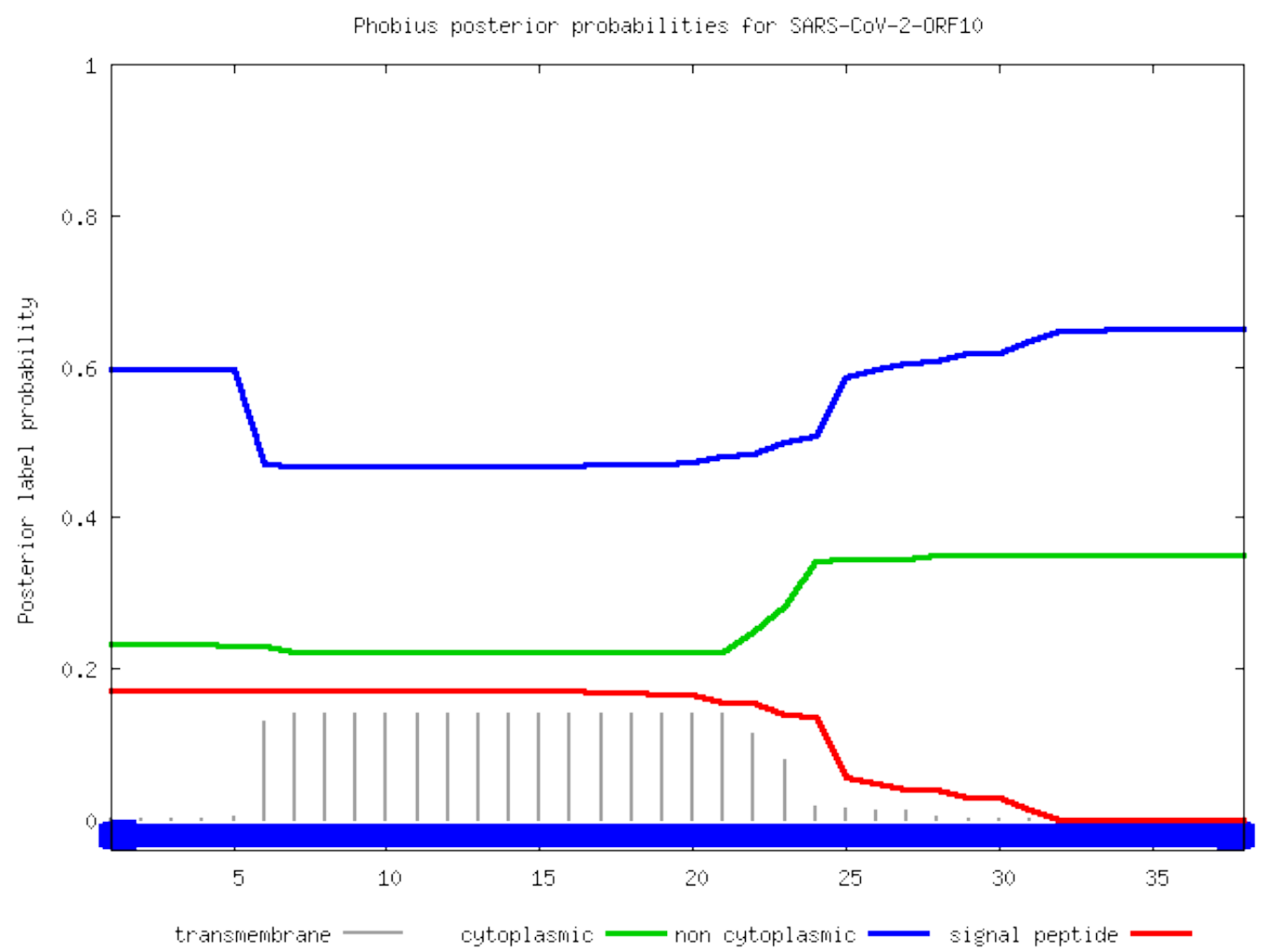

Figure 2. Posterior probability plot for prediction of transmembrane topology and signal peptides for the wild-type SARS-CoV-2 ORF10.

From the posterior probability distributions, it was observed that the SARS-CoV-2 ORF10 protein was entirely non-cytoplasmic (Figure 2).

3.1.2. Unique ORF10 variants and their single and co-occurring mutations

The amino acid residue positions of SARS-CoV-2 ORF10 single-mutations in each continent are listed in Table 2. 
Table 2: Continent-wise amino acid positions of single-mutations in SARS-CoV-2 ORF10 unique variants

\begin{tabular}{cccccc}
\hline \multicolumn{5}{c}{ Continent-wise residue positions of single-mutations } \\
\hline Africa & Asia & Europe & North America & Oceania & South America \\
3 & 6 & 3 & $1-38$ (except 18th) & 3 & 3 \\
5 & 7 & 6 & & 4 & 19 \\
7 & 8 & 13 & 8 & 23 \\
10 & 9 & 23 & 10 & 28 \\
23 & 10 & 30 & 17 & 35 \\
24 & 13 & 31 & 22 & \\
28 & 14 & & 23 & \\
30 & 22 & & 24 & \\
31 & 23 & & 28 & \\
33 & 24 & & 30 & \\
37 & 30 & & 37 & \\
38 & 31 & & 38 & \\
& 33 & & & \\
& 35 & &
\end{tabular}

It was noticed that except for the residue in position 18, all amino acids at each position from 1 to 38 possessed point-missense mutations (Table 2). The common residue was S23, where two mutations S23F and S23P were found across all six continents. A total of eight common residue positions with mutations were observed in Asia and Africa, whereas several common residual mutation positions were associated with Asia and Oceania.

The predicted effects of pathogenicity for each mutation are listed in Table 3. Our data revealed that among a total of 128 mutations, 72 were neutral, and 53 deleterious with regards to pathogenicity. 
Table 3: The predicted effect of ORF10 single mutations on pathogenicity

\begin{tabular}{|c|c|c|c|c|c|c|c|}
\hline \multicolumn{8}{|c|}{ Predicted effect of mutations } \\
\hline & \multicolumn{2}{|c|}{ Percentage of Pathogenicity } & \multirow[b]{2}{*}{ Predcited Type } & \multirow[b]{2}{*}{ Mutation } & \multicolumn{2}{|c|}{ Percentage of Pathogenicity } & \multirow[b]{2}{*}{ Predcited Type } \\
\hline Mutation & PredictSNP & PhD-SNP & & & PredictSNP & PhD-SNP & \\
\hline V33A & $83 \%$ & $66 \%$ & Neutral & N22H & $83 \%$ & $51 \%$ & Neutral \\
\hline V33D & $87 \%$ & $77 \%$ & Deleterious & $\mathrm{N} 22 \mathrm{I}$ & $87 \%$ & $61 \%$ & Deleterious \\
\hline V33F & $83 \%$ & $45 \%$ & Neutral & $\mathrm{N} 22 \mathrm{~K}$ & $87 \%$ & $77 \%$ & Deleterious \\
\hline V33I & $83 \%$ & $78 \%$ & Neutral & $\mathrm{N} 22 \mathrm{~S}$ & $83 \%$ & $68 \%$ & Neutral \\
\hline $\mathrm{D} 31 \mathrm{G}$ & $87 \%$ & $73 \%$ & Deleterious & $\mathrm{N} 22 \mathrm{~T}$ & $87 \%$ & $61 \%$ & Deleterious \\
\hline $\mathrm{D} 31 \mathrm{H}$ & $87 \%$ & $58 \%$ & Deleterious & $\mathrm{N} 22 \mathrm{Y}$ & $87 \%$ & $73 \%$ & Deleterious \\
\hline D31N & $87 \%$ & $68 \%$ & Deleterious & $\mathrm{I} 4 \mathrm{~L}$ & $83 \%$ & $78 \%$ & Neutral \\
\hline $\mathrm{D} 31 \mathrm{~V}$ & $87 \%$ & $82 \%$ & Deleterious & $\mathrm{I} 4 \mathrm{~T}$ & $83 \%$ & $55 \%$ & Neutral \\
\hline D31Y & $87 \%$ & $86 \%$ & Deleterious & $\mathrm{I} 4 \mathrm{~V}$ & $83 \%$ & $83 \%$ & Neutral \\
\hline T38A & $83 \%$ & $78 \%$ & Neutral & R20G & $87 \%$ & $58 \%$ & Deleterious \\
\hline T38I & $83 \%$ & $55 \%$ & Neutral & R20I & $83 \%$ & $68 \%$ & Neutral \\
\hline $\mathrm{L} 37 \mathrm{~F}$ & $83 \%$ & $83 \%$ & Neutral & $\mathrm{R} 20 \mathrm{~K}$ & $83 \%$ & $68 \%$ & Neutral \\
\hline $\mathrm{L} 37 \mathrm{H}$ & $83 \%$ & $58 \%$ & Neutral & $\mathrm{R} 20 \mathrm{~T}$ & $83 \%$ & $51 \%$ & Neutral \\
\hline L37I & $83 \%$ & $83 \%$ & Neutral & M21I & $83 \%$ & $51 \%$ & Neutral \\
\hline L37P & $87 \%$ & $61 \%$ & Deleterious & M21K & $87 \%$ & $82 \%$ & Deleterious \\
\hline L37R & $87 \%$ & $61 \%$ & Deleterious & M21L & $87 \%$ & $58 \%$ & Deleterious \\
\hline $\mathrm{Y} 3 \mathrm{C}$ & $83 \%$ & $58 \%$ & Neutral & M21T & $87 \%$ & $73 \%$ & Deleterious \\
\hline $\mathrm{Y} 3 \mathrm{H}$ & $83 \%$ & $72 \%$ & Neutral & M21V & $83 \%$ & $58 \%$ & Neutral \\
\hline N5D & $83 \%$ & $66 \%$ & Neutral & N25D & $83 \%$ & $45 \%$ & Neutral \\
\hline N5K & $83 \%$ & $55 \%$ & Neutral & N25G & $87 \%$ & $59 \%$ & Deleterious \\
\hline N5S & $83 \%$ & $66 \%$ & Neutral & N25S & $83 \%$ & $55 \%$ & Neutral \\
\hline N5Y & $87 \%$ & $68 \%$ & Deleterious & Y26C & $87 \%$ & $68 \%$ & Deleterious \\
\hline $\mathrm{R} 24 \mathrm{C}$ & $87 \%$ & $61 \%$ & Deleterious & Y26F & $83 \%$ & $68 \%$ & Neutral \\
\hline $\mathrm{R} 24 \mathrm{H}$ & $83 \%$ & $51 \%$ & Neutral & $\mathrm{Y} 26 \mathrm{H}$ & $83 \%$ & $58 \%$ & Neutral \\
\hline $\mathrm{R} 24 \mathrm{~L}$ & $87 \%$ & $61 \%$ & Deleterious & $\mathrm{I} 27 \mathrm{~K}$ & $87 \%$ & $86 \%$ & Deleterious \\
\hline $\mathrm{R} 24 \mathrm{~S}$ & $83 \%$ & $58 \%$ & Neutral & $\mathrm{I} 27 \mathrm{~L}$ & $83 \%$ & $51 \%$ & Neutral \\
\hline $\mathrm{S} 23 \mathrm{~F}$ & $87 \%$ & $58 \%$ & Deleterious & $\mathrm{I} 27 \mathrm{M}$ & $87 \%$ & $58 \%$ & Deleterious \\
\hline $\mathrm{S} 23 \mathrm{P}$ & $83 \%$ & $51 \%$ & Neutral & $\mathrm{I} 27 \mathrm{R}$ & $87 \%$ & $86 \%$ & Deleterious \\
\hline P10L & $87 \%$ & $86 \%$ & Deleterious & $\mathrm{I} 27 \mathrm{~T}$ & $87 \%$ & $77 \%$ & Deleterious \\
\hline P10Q & $7 \%$ & $73 \%$ & Deleterious & $\mathrm{I} 27 \mathrm{~V}$ & $83 \%$ & $66 \%$ & Neutral \\
\hline P10S & $83 \%$ & $51 \%$ & Neutral & D31G & $87 \%$ & $73 \%$ & Deleterious \\
\hline P10T & $87 \%$ & $68 \%$ & Deleterious & D31H & $87 \%$ & $58 \%$ & Deleterious \\
\hline F7C & $87 \%$ & $59 \%$ & Deleterious & D31N & $87 \%$ & $68 \%$ & Deleterious \\
\hline F7L & $83 \%$ & $55 \%$ & Neutral & D31V & $87 \%$ & $82 \%$ & Deleterious \\
\hline F7S & $83 \%$ & $55 \%$ & Neutral & D31Y & $87 \%$ & $86 \%$ & Deleterious \\
\hline V6A & $83 \%$ & $78 \%$ & Neutral & M1G & $83 \%$ & $72 \%$ & Neutral \\
\hline V6F & $83 \%$ & $55 \%$ & Neutral & G2D & $83 \%$ & $68 \%$ & Neutral \\
\hline V6I & $83 \%$ & $78 \%$ & Neutral & G2L & $83 \%$ & $58 \%$ & Neutral \\
\hline Y14C & $87 \%$ & $68 \%$ & Deleterious & $\mathrm{F} 11 \mathrm{~L}$ & $87 \%$ & $73 \%$ & Deleterious \\
\hline $\mathrm{Y} 14 \mathrm{~F}$ & $83 \%$ & $72 \%$ & Neutral & F11S & $87 \%$ & $61 \%$ & Deleterious \\
\hline $\mathrm{Y} 14 \mathrm{H}$ & $83 \%$ & $55 \%$ & Neutral & $\mathrm{T} 12 \mathrm{~A}$ & $83 \%$ & $58 \%$ & Neutral \\
\hline $\mathrm{A} 28 \mathrm{P}$ & $87 \%$ & $73 \%$ & Deleterious & $\mathrm{T} 12 \mathrm{M}$ & $87 \%$ & $73 \%$ & Deleterious \\
\hline A28S & $83 \%$ & $51 \%$ & Neutral & $\mathrm{S} 15 \mathrm{C}$ & $83 \%$ & $51 \%$ & Neutral \\
\hline $\mathrm{A} 28 \mathrm{~V}$ & $83 \%$ & $55 \%$ & Neutral & S15G & $83 \%$ & $58 \%$ & Neutral \\
\hline V30A & $83 \%$ & $55 \%$ & Neutral & L16P & $87 \%$ & $86 \%$ & Deleterious \\
\hline V30I & $83 \%$ & $78 \%$ & Neutral & L17F & $83 \%$ & $72 \%$ & Neutral \\
\hline V30L & $83 \%$ & $51 \%$ & Neutral & L17P & $87 \%$ & $86 \%$ & Deleterious \\
\hline F35C & $87 \%$ & $59 \%$ & Deleterious & $\mathrm{C} 19 \mathrm{~F}$ & $87 \%$ & $59 \%$ & Deleterious \\
\hline F35S & $83 \%$ & $51 \%$ & Neutral & $\mathrm{S} 23 \mathrm{~F}$ & $87 \%$ & $58 \%$ & Deleterious \\
\hline A8D & $87 \%$ & $77 \%$ & Deleterious & $\mathrm{S} 23 \mathrm{P}$ & $83 \%$ & $51 \%$ & Neutral \\
\hline $\mathrm{A} 8 \mathrm{G}$ & $83 \%$ & $68 \%$ & Neutral & Q29H & $83 \%$ & $55 \%$ & Neutral \\
\hline $\mathrm{A} 8 \mathrm{P}$ & $87 \%$ & $73 \%$ & Deleterious & Q29L & $87 \%$ & $61 \%$ & Deleterious \\
\hline A8S & $83 \%$ & $55 \%$ & Neutral & Q29R & $87 \%$ & $58 \%$ & Deleterious \\
\hline $\mathrm{A} 8 \mathrm{~V}$ & $83 \%$ & $58 \%$ & Neutral & V32A & $83 \%$ & $66 \%$ & Neutral \\
\hline F9L & $87 \%$ & $61 \%$ & Deleterious & V32I & $83 \%$ & $78 \%$ & Neutral \\
\hline F9S & $87 \%$ & $58 \%$ & Deleterious & V32L & $83 \%$ & $55 \%$ & Neutral \\
\hline F9Y & $83 \%$ & $51 \%$ & Neutral & N34D & $83 \%$ & $66 \%$ & Neutral \\
\hline I13L & $83 \%$ & $55 \%$ & Neutral & N34Y & $87 \%$ & $73 \%$ & Deleterious \\
\hline $\mathrm{I} 13 \mathrm{M}$ & $83 \%$ & $51 \%$ & Neutral & N36K & $83 \%$ & $51 \%$ & Neutral \\
\hline $\mathrm{I} 13 \mathrm{~T}$ & $87 \%$ & $61 \%$ & Deleterious & N36S & $83 \%$ & $72 \%$ & Neutral \\
\hline $\mathrm{I} 13 \mathrm{~V}$ & $83 \%$ & $68 \%$ & Neutral & $\mathrm{T} 38 \mathrm{~A}$ & $83 \%$ & $78 \%$ & Neutral \\
\hline N22D & $83 \%$ & $58 \%$ & Neutral & T38I & $83 \%$ & $55 \%$ & Neutral \\
\hline $\mathrm{N} 22 \mathrm{~F}$ & $83 \%$ & $55 \%$ & Neutral & & & & \\
\hline
\end{tabular}

Among a total of 37 residue positions with single mutations, the residue position $1,2,11,12,15,16,20,21,25,26,27,29,32,34$, and 36 were unique in North America. Among all these residue positions, only the mutations at positions 11 and 16 in ORF10 variants in North America were predicted to be deleterious.

Furthermore, co-mutations in the SARS-CoV-2 ORF10 in some geo-locations were listed in Table 4. The highest number of simultaneous mutations (at 14 positions among the total of 38 amino acid residues) in ORF10 was noticed in a SARS-CoV-2 variant from 
Russia on March 21, 2020. Interestingly, no report of this kind of co-occurrent mutation globally, hence denoted by localized index 1 .

Table 4: Co-occurrent mutations in the ORF10 variants in various geo-locations

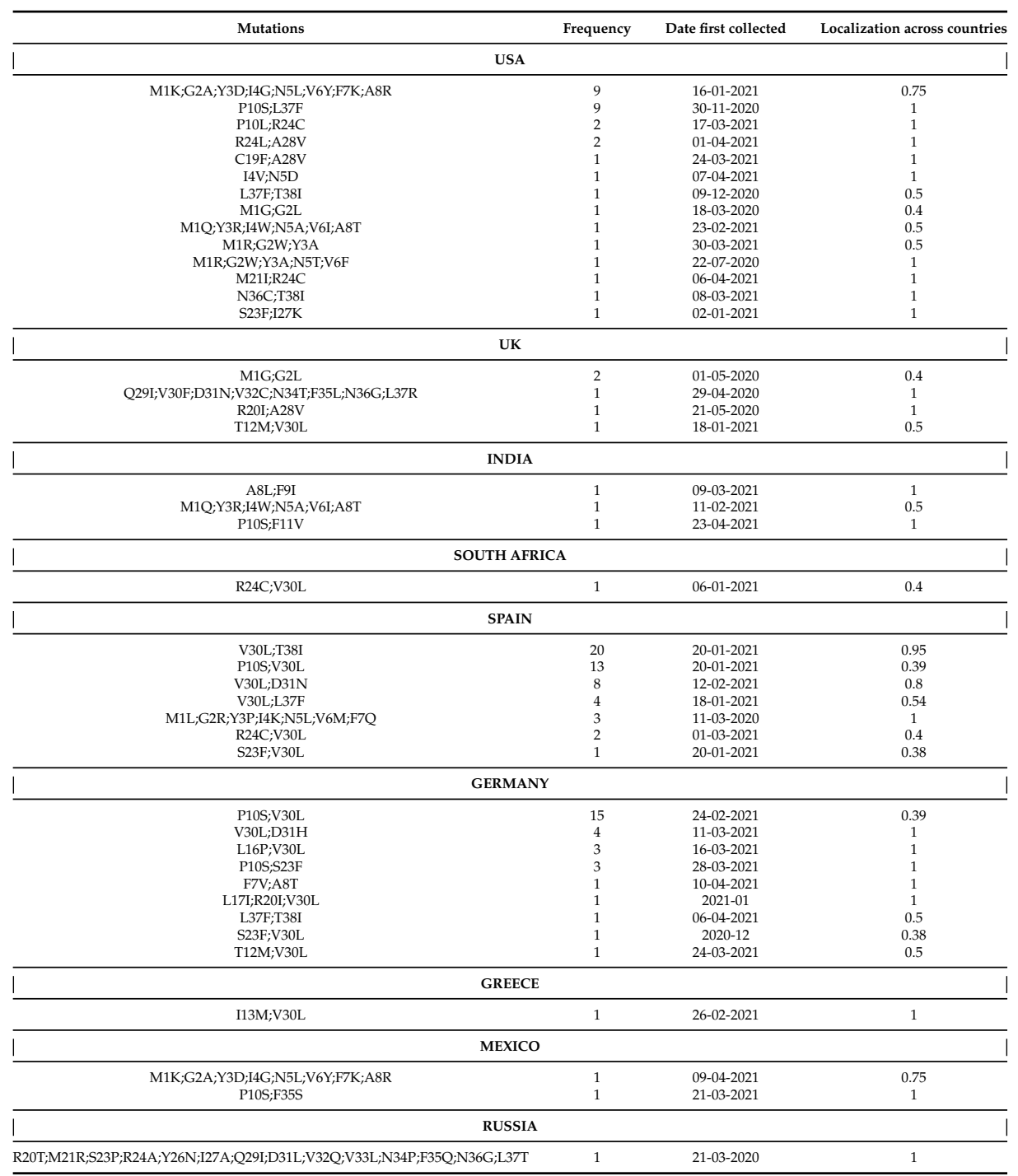

Several co-mutations in SARS-CoV-2 ORF10 variants have been reported in the USA, UK, India, South Africa, Spain, Germany, Greece, Mexico, and Russia. The most co-occurrent mutations (V30L, T38I, first reported on January 20, 2021) were found in 20 infected patients in Spain. The US first detected and reported co-occurrent mutations M1K, G2A, Y3D, I4G, N5L, V6Y, F7K, and A8R on January 16, 2021 from 9 infected patients, and later on in April 9, 2021 Germany reported its first case from one patient (Table 4). It is worth knowing that no single mutation at the residue positions 1 and 2 have been reported, and none of the co-mutations among M1K, G2A, Y3D, I4G, N5L, V6Y, F7K, and A8R were reported as a single mutation in the ORF10 variants. Double co-mutations P10S; V301 were reported on January 20, 2021 in Spain on January 20, 2021, and somewhere late February 24, 2021, in Germany. Surprisingly, the pathogenetic effects were predicted to be neutral (Table 3). It was noticed that V30L, one of the most common mutations in ORF10, was co-occurring with most of the other co-mutations. It appears that co-mutations in the SARS-CoV-2 ORF10 variants is an emerging trend. 


\subsection{Intrinsic disorder regions of SARS-CoV-2 ORF10 variants}

The per-residue disorder profiles for 138 unique SARS-CoV-2 ORF10 variants (variants are too short for disorder analysis) and 35 co-occurring mutations are presented in Figure 3. Figure 3A shows that the intrinsic disorder predisposition of ORF10 is noticeably affected by single mutations. Mutations present the largest effects at the $\mathrm{N}$ - and C-terminal regions, where the disorder predisposition can vary from 0.4 to 0.8 and from 0.55 to 0.9 for the $\mathrm{N}$ - and C-termini, respectively, and for a region centered at residue 25 , where the disorder score can change from 0.059 to 0.27 . Figure $3 \mathrm{~B}$ compares the outputs of PONDR-VSL2 for 138 unique ORF10 variants with the results generated for these proteins by one of the most conservative predictors of intrinsic disorder, and IUPred_short [50].
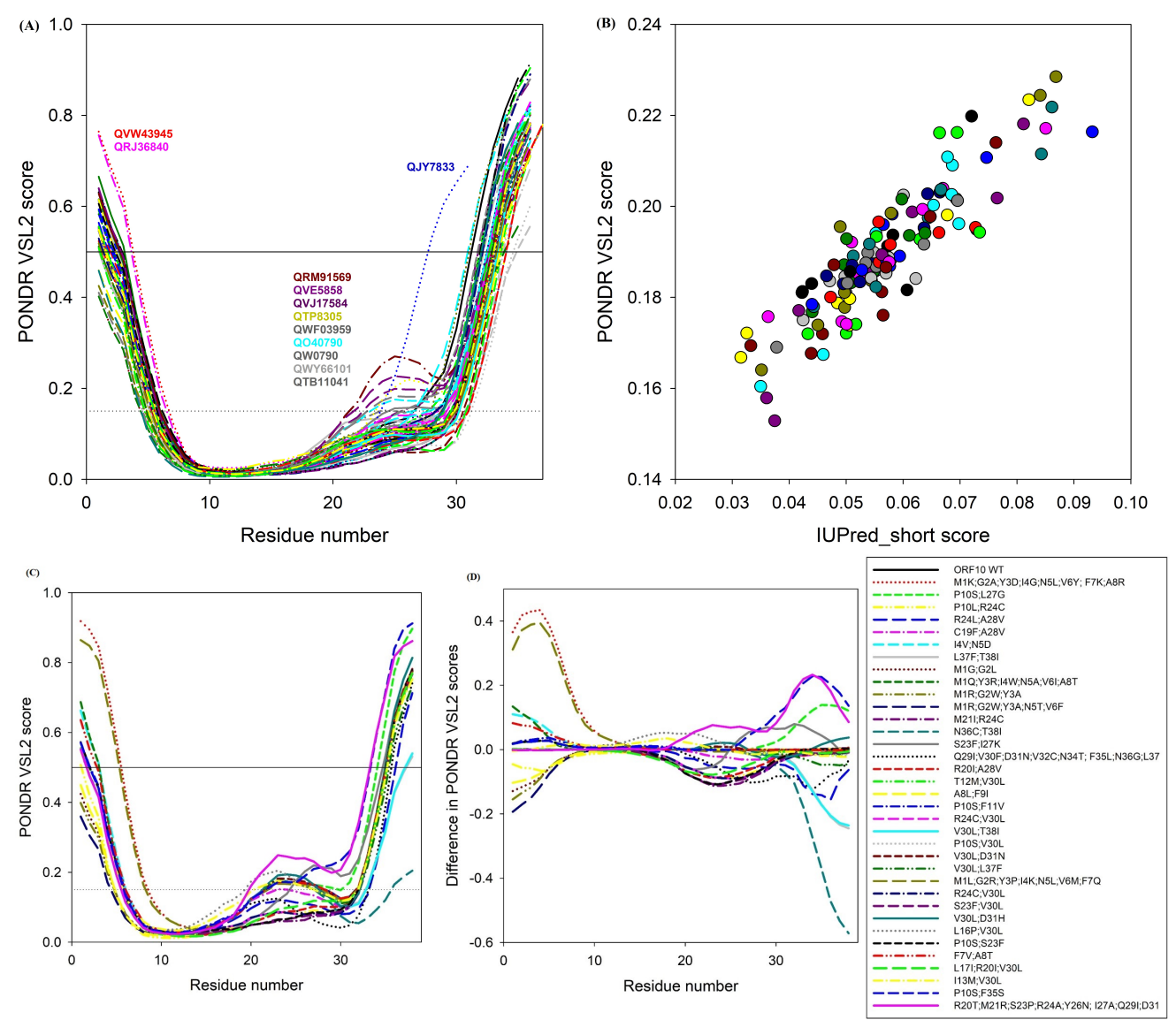

Figure 3. (A): One shows per-residue disorder profiles for 138 SARS-CoV-2 ORF10 variants, (B): Correlation between the outputs of two commonly used disorder predictors, $(C)$ shows how disorder propensity is changed in ORF10 variants with co-occurring mutations, and (D) difference spectra calculated by subtracting off the per-residue disorder scores of wild type ORF10 from the per-residue disorder scores of corresponding mutants.

Here, we investigate the effects of point mutations on the overall disorder score for the entire protein. This analysis showed that although the disorder score values generated by IUPred_short are noticeably smaller than the corresponding PONDR-VSL2 data, the scales of changes introduced by single mutations in intrinsic disorder predisposition of ORF10 as evaluated by IUPred (from 0.03 to 0.095 ) are comparable to that generated by PONDR VSL2 (from 0.15 to 0.23 ). Furthermore, both predictors mostly agree on the direction of the changes introduced in the global disorder propensity of this protein by single mutations. Figure $3 \mathrm{C}$ shows how disorder propensity is changed in ORF10 
variants with co-occurring mutations. Noticeably, the effects of co-occurring mutants are qualitatively similar to those of single mutations, as most variability is observed at the disorder predisposition of $\mathrm{N}$ - and C-terminal regions and a region centered at residue 25 . However, in the case of co-occurring mutants, scales of changes at the terminal regions are noticeably larger (the disorder predisposition can vary from 0.35 to 0.92 and from 0.2 to 0.9 for the $\mathrm{N}$ - and C-termini, respectively). Finally, to simplify comparison of the effect of co-occurring mutations on the disorder predisposition of ORF10, Figure 3D shows "difference spectra" calculated by subtracting of the per-residue disorder scores of wild type ORF10 from the per-residue disorder scores of corresponding mutants. In this plot, intensities of the resulting "bands" reflect the scale of changes, whereas their sign, reflects the mutation-induced increase or decrease (positive or negative values, respectively) in local disorder propensity. Figure 3D shows that most of the co-occurring mutations increase the disorder predisposition of the N-terminal region of ORF10, whereas many such mutations decrease the disorder propensity of the region centered at residue 25, and most of the mutations do not affect disorder predisposition of the C-terminal region.

\subsection{Predicting secondary structures of ORF10 protein variants with co-mutations}

The SARS-CoV-2 ORF10 protein was found to interact with few of the human proteins such as CUL2, ELOB, ELOC, MAP7D1, PPT1, RBX1, THTPA, TIMM8B, and ZYG11B [33]. A detailed summary of the tissue and cellular expression patterns of SARS-CoV-2 ORF10 interacting human proteins, based on transcriptomics and antibodybased proteomics, are presented in Figure 4. The ORF10 of SARS-CoV-2 interacts with members of the Cullin ubiquitin ligase CUL2 ${ }^{Z Y G 11 B}$ complex. Interestingly, among the genes ZYG11B scored the highest with regards to ORF10 interactome, which confirmed a direct interaction between ORF10 and ZYG11B [32].

\begin{tabular}{|c|c|c|c|c|c|c|c|}
\hline Gene name ${ }^{i}$ & 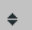 & Covid-19 bait' & $\Leftrightarrow$ & Tissue specificity' & Tissue expression ${ }^{i}$ & Blood specificity' & Subcellular location (main) \\
\hline CUL2 & & SARS-CoV2 orf10 & & Low tissue specificity & $\square$ & Low immune cell specificity & Nucleoplasm \\
\hline ELOB & & SARS-CoV2 orf 10 & & Low tissue specificity & $\square$ & Low immune cell specificity & N/A \\
\hline ELOC & & SARS-CoV2 orf10 & & Low tissue specificity & N/A & Low immune cell specificity & Vesicles \\
\hline MAP7D1 & & SARS-CoV2 orf10 & & $\begin{array}{l}\text { Tissue enhanced (skeletal } \\
\text { muscle) }\end{array}$ & $\square$ & $\begin{array}{l}\text { Immune cell enhanced } \\
\text { (neutrophil) }\end{array}$ & Microtubules, Cytosol \\
\hline PPT1 & & SARS-CoV2 orf 10 & & Low tissue specificity & $\square$ & Low immune cell specificity & Golgi apparatus, Vesicles \\
\hline RBX1 & & SARS-CoV2 orf10 & & Low tissue specificity & N/A & Low immune cell specificity & Nucleoplasm \\
\hline THTPA & & SARS-CoV2 orf10 & & Low tissue specificity & $\square$ & Low immune cell specificity & Nucleoplasm \\
\hline TIMM8B & & SARS-CoV2 orf10 & & Low tissue specificity & $\square$ & Low immune cell specificity & $\mathrm{N} / \mathrm{A}$ \\
\hline ZYG11B & & SARS-CoV2 orf10 & & $\begin{array}{l}\text { Tissue enhanced (skeletal } \\
\text { muscle) }\end{array}$ & & Low immune cell specificity & $\begin{array}{l}\text { Golgi apparatus, Intermediate } \\
\text { filaments }\end{array}$ \\
\hline
\end{tabular}

Figure 4. A snapshot summary of the tissue and cellular expression patterns of SARS-CoV-2 ORF10 interacting human proteins, based on transcriptomics and antibody-based proteomics.

The SARS-CoV-2 ORF10 interacting with human proteins CUL2, ELOB, PPT1, THTPA, and TIMM8B were expressed in various tissues, including the lung. SARSCoV-2 ORF10 contains an $\alpha$-helical region (amino acid residue positions 3 to 20), which may interact and form a complex with CUL2 ${ }^{Z Y G 11 B}$ [32]. It was furthermore reported that by forming a complex with $C U L 2^{Z Y G 11 B}$, ORF10 hijacks it for ubiquitination and degradation of restriction factors, or alternatively, may binds to the $\mathrm{N}$-terminal glycine in ORF10 to target it for degradation [32,33].

The secondary structures of nine ORF10 variants including the wild ORF10 (YP_009725255) with several co-mutations were predicted (Figure 5). The $\alpha$-helix secondary structural amino acid (aa) residue position 3 to 20 were invariant for the six ORF variants with co-mutations as mentioned in panel (4) to (9) of Figure 5, although the reliability indices were changed for the $\alpha$-helix region [32]. 

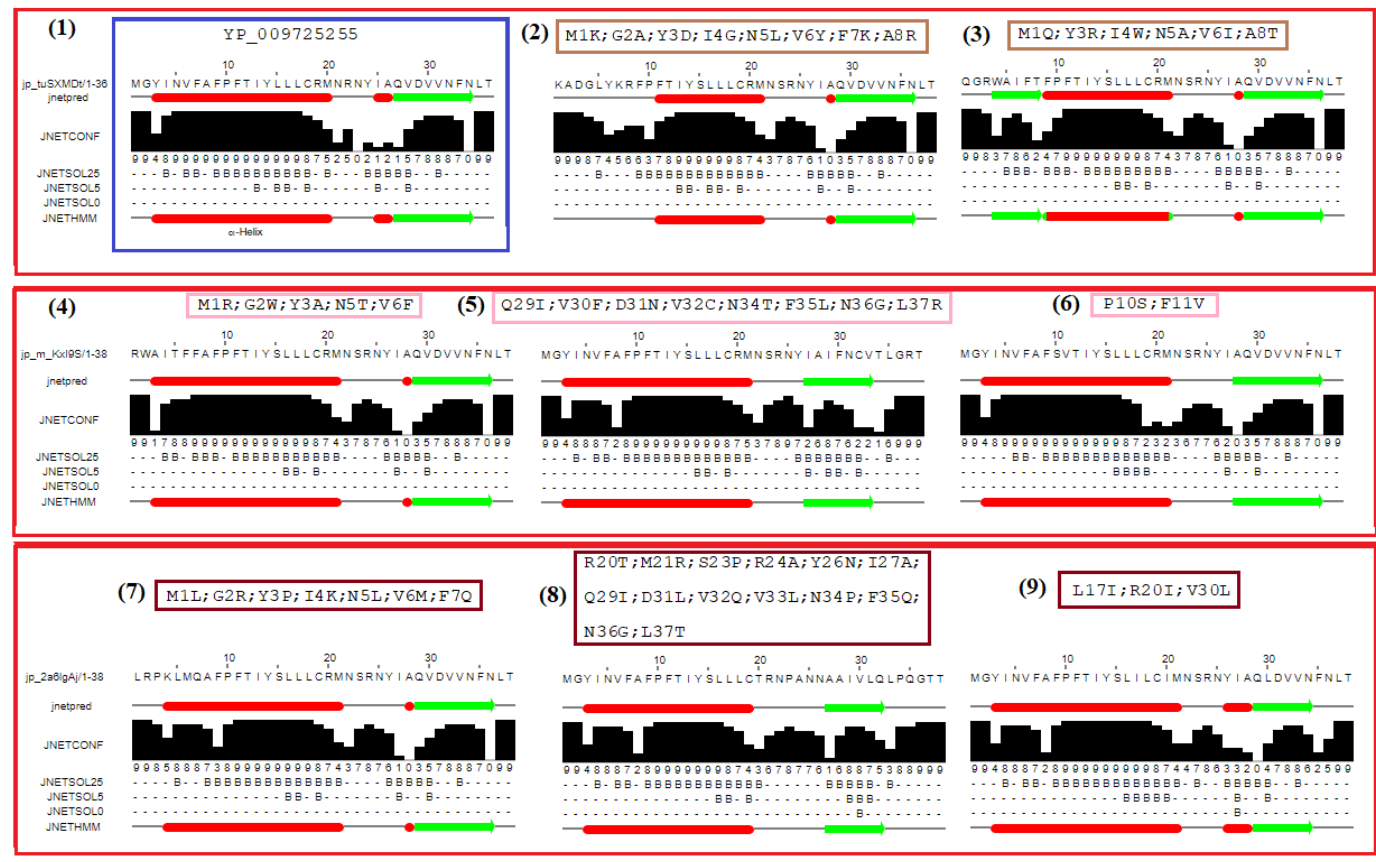

Figure 5. Predicted secondary structure of ORF10 variants with co-mutations.

Furthermore, it was observed that the $\alpha$-helix amino acid residue positions (aa positions 3-20) got changed to 11-21 and 9-21 due to the co-mutations M1K, G2A, Y3D, I4G, N5L, V6Y, F7K, A8R, and M1Q, Y3R, I4W, N5A, V6I, A8T in two different ORF10 variants, respectively. Therefore, due to these two sets of co-mutations, SARS-CoV-2 ORF10 interactions with the CUL2 ${ }^{Z Y G 11 B}$ complex might be affected. Due to the amino acid residue positional changes in the secondary structure in the ORF10 variants (other than the reference ORF10 (YP_009725255)), these ORF10 variants with human proteins other than the CUL2 ${ }^{Z Y G 11 B}$ complex is likely to be affected.

\subsection{Amino acid frequency distribution across the unique ORF10 variants and associated clusters}

The frequency of each amino acid in the 140 unique ORF10 sequences are presented in Tables 5 and 6. Also, Figure 6(A) shows the amino acid frequency vector of each unique ORF19 sequence. It was noticed that glutamic acid (E) and tryptophan (W) were absent in each ORF10 variant. Among 140 unique ORF10 variants, lysine (K) was present with a single frequency only in seven North American ORF10 variants (QTD22916.1, QLA48060.1, QWY66101.1, QVO40425.1, QTP28305.1, QSL79091.1, and QRM91569.1). 
Table 5: Frequency distribution of amino acids over the 140 unique SARS-CoV-2 ORF10 variants (from QKM75696.1 to QWN58574.1)

\begin{tabular}{|c|c|c|c|c|c|c|c|c|c|c|c|c|c|c|c|c|c|c|c|c|}
\hline ORF10 & $\mathbf{A}$ & $\mathbf{R}$ & $\mathbf{N}$ & D & $\mathrm{C}$ & $\mathbf{Q}$ & E & G & $\mathbf{H}$ & I & $\mathbf{L}$ & K & $\mathbf{M}$ & F & $\mathbf{P}$ & $\mathrm{S}$ & $\mathrm{T}$ & $\mathbf{W}$ & $\mathbf{Y}$ & $\mathbf{V}$ \\
\hline QKM75696.1 & 2 & 2 & 4 & 1 & 1 & 1 & 0 & 0 & 0 & 2 & 4 & 0 & 1 & 3 & 1 & 2 & 2 & 0 & 2 & 3 \\
\hline QKKG88643.1 & 2 & 2 & 5 & 1 & 1 & 1 & 0 & 1 & 0 & 3 & 5 & 0 & 1 & 4 & 1 & 2 & 2 & 0 & 3 & 4 \\
\hline QVJ13930.1 & 2 & 2 & 5 & 2 & 1 & 1 & 0 & 0 & 0 & 3 & 4 & 0 & 2 & 4 & 1 & 2 & 2 & 0 & 3 & 4 \\
\hline QWE67724.1 & 2 & 2 & 5 & 1 & 2 & 1 & 0 & 1 & 0 & 3 & 4 & 0 & 2 & 4 & 1 & 2 & 2 & 0 & 2 & 4 \\
\hline QRJ36840.1 & 2 & 2 & 5 & 1 & 1 & 1 & 0 & 1 & 1 & 3 & 4 & 0 & 2 & 4 & 1 & 2 & 2 & 0 & 2 & 4 \\
\hline QQY03084.1 & 2 & 2 & 4 & 1 & 1 & 1 & 0 & 1 & 0 & 3 & 4 & 0 & 2 & 4 & 1 & 2 & 2 & 0 & 3 & 4 \\
\hline QRG22086.1 & 2 & 2 & 4 & 2 & 1 & 1 & 0 & 1 & 0 & 3 & 4 & 0 & 2 & 4 & 1 & 2 & 2 & 0 & 3 & 4 \\
\hline QTD22916.1 & 2 & 2 & 4 & 1 & 1 & 1 & 0 & 1 & 0 & 3 & 4 & 1 & 2 & 4 & 1 & 2 & 2 & 0 & 3 & 4 \\
\hline QUL69971.1 & 2 & 2 & 5 & 1 & 1 & 1 & 0 & 1 & 0 & 3 & 4 & 0 & 2 & 2 & 0 & 2 & 2 & 0 & 3 & 3 \\
\hline QWB85197.1 & 2 & 2 & 5 & 1 & 1 & 1 & 0 & 1 & 0 & 3 & 4 & 0 & 2 & 5 & 1 & 2 & 2 & 0 & 3 & 3 \\
\hline QWJ83116.1 & 2 & 2 & 5 & 1 & 1 & 1 & 0 & 1 & 0 & 4 & 4 & 0 & 2 & 4 & 1 & 2 & 2 & 0 & 3 & 3 \\
\hline QSE09446.1 & 2 & 2 & 5 & 1 & 2 & 1 & 0 & 1 & 0 & 3 & 4 & 0 & 2 & 3 & 1 & 2 & 2 & 0 & 3 & 4 \\
\hline QWZ00470.1 & 2 & 2 & 5 & 1 & 1 & 1 & 0 & 1 & 0 & 3 & 5 & 0 & 2 & 4 & 0 & 2 & 2 & 0 & 3 & 4 \\
\hline QTP26076.1 & 3 & 2 & 5 & 1 & 1 & 1 & 0 & 1 & 0 & 3 & 4 & 0 & 2 & 4 & 1 & 2 & 1 & 0 & 3 & 4 \\
\hline QWK62875.1 & 2 & 2 & 5 & 1 & 1 & 1 & 0 & 1 & 0 & 3 & 4 & 0 & 3 & 4 & 1 & 2 & 1 & 0 & 3 & 4 \\
\hline QVG57396.1 & 2 & 2 & 5 & 1 & 2 & 1 & 0 & 1 & 0 & 3 & 4 & 0 & 2 & 4 & 1 & 2 & 2 & 0 & 2 & 4 \\
\hline QRA60944.1 & 2 & 2 & 5 & 1 & 1 & 1 & 0 & 1 & 0 & 3 & 4 & 0 & 2 & 5 & 1 & 2 & 2 & 0 & 2 & 4 \\
\hline QWE68295.1 & 2 & 2 & 5 & 1 & 1 & 1 & 0 & 1 & 1 & 3 & 4 & 0 & 2 & 4 & 1 & 2 & 2 & 0 & 2 & 4 \\
\hline QWT58729.1 & 2 & 2 & 5 & 1 & 2 & 1 & 0 & 1 & 0 & 3 & 4 & 0 & 2 & 4 & 1 & 1 & 2 & 0 & 3 & 4 \\
\hline QWM42669.1 & 2 & 2 & 5 & 1 & 1 & 1 & 0 & 2 & 0 & 3 & 4 & 0 & 2 & 4 & 1 & 1 & 2 & 0 & 3 & 4 \\
\hline QWS64226.1 & 2 & 2 & 5 & 1 & 1 & 1 & 0 & 1 & 0 & 3 & 3 & 0 & 2 & 5 & 1 & 2 & 2 & 0 & 3 & 4 \\
\hline QVJ47956.1 & 2 & 1 & 5 & 1 & 1 & 1 & 0 & 2 & 0 & 3 & 4 & 0 & 2 & 4 & 1 & 2 & 2 & 0 & 3 & 4 \\
\hline QVW78101.1 & 2 & 1 & 5 & 1 & 1 & 1 & 0 & 1 & 0 & 4 & 4 & 0 & 2 & 4 & 1 & 2 & 2 & 0 & 3 & 4 \\
\hline QLA48060.1 & 2 & 1 & 5 & 1 & 1 & 1 & 0 & 1 & 0 & 3 & 4 & 1 & 2 & 4 & 1 & 2 & 2 & 0 & 3 & 4 \\
\hline QVO98764.1 & 2 & 2 & 5 & 1 & 1 & 1 & 0 & 1 & 0 & 4 & 4 & 0 & 1 & 4 & 1 & 2 & 2 & 0 & 3 & 4 \\
\hline QWY66101.1 & 2 & 2 & 5 & 1 & 1 & 1 & 0 & 1 & 0 & 3 & 4 & 1 & 1 & 4 & 1 & 2 & 2 & 0 & 3 & 4 \\
\hline QWQ05246.1 & 2 & 2 & 5 & 1 & 1 & 1 & 0 & 1 & 0 & 3 & 5 & 0 & 1 & 4 & 1 & 2 & 2 & 0 & 3 & 4 \\
\hline QTS35265.1 & 2 & 2 & 4 & 2 & 1 & 1 & 0 & 1 & 0 & 3 & 4 & 0 & 2 & 4 & 1 & 2 & 2 & 0 & 3 & 4 \\
\hline QVP24786.1 & 2 & 2 & 4 & 1 & 1 & 1 & 0 & 1 & 1 & 3 & 4 & 0 & 2 & 4 & 1 & 2 & 2 & 0 & 3 & 4 \\
\hline QVO85840.1 & 2 & 2 & 4 & 1 & 1 & 1 & 0 & 1 & 0 & 4 & 4 & 0 & 2 & 4 & 1 & 2 & 2 & 0 & 3 & 4 \\
\hline QVO40425.1 & 2 & 2 & 4 & 1 & 1 & 1 & 0 & 1 & 0 & 3 & 4 & 1 & 2 & 4 & 1 & 2 & 2 & 0 & 3 & 4 \\
\hline QWU52456.1 & 2 & 2 & 5 & 1 & 1 & 1 & 0 & 1 & 0 & 3 & 4 & 0 & 2 & 5 & 1 & 1 & 2 & 0 & 3 & 4 \\
\hline QTP28305.1 & 2 & 2 & 5 & 1 & 1 & 1 & 0 & 1 & 0 & 2 & 4 & 1 & 2 & 5 & 1 & 1 & 2 & 0 & 3 & 4 \\
\hline QWS07290.1 & 2 & 2 & 5 & 1 & 1 & 1 & 0 & 1 & 0 & 3 & 4 & 0 & 2 & 4 & 2 & 1 & 2 & 0 & 3 & 4 \\
\hline QWF07009.1 & 2 & 1 & 5 & 1 & 2 & 1 & 0 & 1 & 0 & 3 & 3 & 0 & 2 & 5 & 1 & 2 & 2 & 0 & 3 & 4 \\
\hline QWY19801.1 & 2 & 1 & 5 & 1 & 2 & 1 & 0 & 1 & 0 & 3 & 4 & 0 & 2 & 4 & 1 & 2 & 2 & 0 & 3 & 4 \\
\hline QWX30181.1 & 2 & 1 & 5 & 1 & 1 & 1 & 0 & 1 & 1 & 3 & 4 & 0 & 2 & 4 & 1 & 2 & 2 & 0 & 3 & 4 \\
\hline QWY95666.1 & 2 & 1 & 5 & 1 & 1 & 1 & 0 & 1 & 0 & 3 & 5 & 0 & 2 & 4 & 1 & 2 & 2 & 0 & 3 & 4 \\
\hline QVU00656.1 & 2 & 2 & 4 & 2 & 1 & 1 & 0 & 1 & 0 & 3 & 4 & 0 & 2 & 4 & 1 & 2 & 2 & 0 & 3 & 4 \\
\hline QTA53643.1 & 3 & 2 & 4 & 1 & 1 & 1 & 0 & 2 & 0 & 3 & 4 & 0 & 2 & 4 & 1 & 2 & 2 & 0 & 3 & 3 \\
\hline QUG14309.1 & 2 & 2 & 4 & 1 & 1 & 1 & 0 & 2 & 0 & 3 & 4 & 0 & 2 & 4 & 1 & 2 & 2 & 0 & 3 & 4 \\
\hline QWY70751.1 & 2 & 2 & 5 & 1 & 2 & 1 & 0 & 1 & 0 & 3 & 4 & 0 & 2 & 4 & 1 & 2 & 2 & 0 & 2 & 4 \\
\hline QSJ35636.1 & 2 & 2 & 5 & 1 & 1 & 1 & 0 & 1 & 0 & 3 & 4 & 0 & 2 & 5 & 1 & 2 & 2 & 0 & 2 & 4 \\
\hline QWF03959.1 & 2 & 2 & 5 & 1 & 1 & 1 & 0 & 1 & 1 & 3 & 4 & 0 & 2 & 4 & 1 & 2 & 2 & 0 & 2 & 4 \\
\hline BCY15724.1 & 2 & 2 & 3 & 0 & 1 & 0 & 0 & 1 & 0 & 3 & 3 & 0 & 2 & 3 & 1 & 2 & 1 & 0 & 3 & 1 \\
\hline QWT72678.1 & 2 & 2 & 5 & 1 & 1 & 0 & 0 & 1 & 1 & 3 & 4 & 0 & 2 & 4 & 1 & 2 & 2 & 0 & 3 & 4 \\
\hline QUM42028.1 & 2 & 2 & 5 & 1 & 1 & 0 & 0 & 1 & 0 & 3 & 5 & 0 & 2 & 4 & 1 & 2 & 2 & 0 & 3 & 4 \\
\hline QUX49292.1 & 3 & 2 & 5 & 1 & 1 & 1 & 0 & 1 & 0 & 3 & 4 & 0 & 2 & 4 & 1 & 2 & 2 & 0 & 3 & 3 \\
\hline QWK69365.1 & 2 & 2 & 5 & 1 & 1 & 1 & 0 & 1 & 0 & 4 & 4 & 0 & 2 & 4 & 1 & 2 & 2 & 0 & 3 & 3 \\
\hline QTA74333.1 & 2 & 2 & 5 & 1 & 1 & 1 & 0 & 1 & 0 & 3 & 4 & 0 & 2 & 5 & 1 & 2 & 2 & 0 & 3 & 3 \\
\hline QWY54619.1 & 2 & 2 & 5 & 1 & 1 & 1 & 0 & 1 & 0 & 3 & 5 & 0 & 2 & 4 & 1 & 2 & 2 & 0 & 3 & 3 \\
\hline QUV26065.1 & 3 & 2 & 5 & 1 & 1 & 1 & 0 & 1 & 0 & 3 & 4 & 0 & 2 & 4 & 1 & 2 & 2 & 0 & 3 & 3 \\
\hline QWF05003.1 & 2 & 2 & 5 & 1 & 1 & 1 & 0 & 1 & 0 & 4 & 4 & 0 & 2 & 4 & 1 & 2 & 2 & 0 & 3 & 3 \\
\hline QUP00476.1 & 2 & 2 & 5 & 1 & 1 & 1 & 0 & 1 & 0 & 3 & 5 & 0 & 2 & 4 & 1 & 2 & 2 & 0 & 3 & 3 \\
\hline QTJ93574.1 & 3 & 2 & 5 & 1 & 1 & 1 & 0 & 1 & 0 & 3 & 4 & 0 & 2 & 4 & 1 & 2 & 2 & 0 & 3 & 3 \\
\hline QWN49685.1 & 2 & 2 & 5 & 2 & 1 & 1 & 0 & 1 & 0 & 3 & 4 & 0 & 2 & 4 & 1 & 2 & 2 & 0 & 3 & 3 \\
\hline QVO91006.1 & 2 & 2 & 5 & 1 & 1 & 1 & 0 & 1 & 0 & 3 & 4 & 0 & 2 & 5 & 1 & 2 & 2 & 0 & 3 & 3 \\
\hline QVV18442.1 & 2 & 2 & 5 & 1 & 1 & 1 & 0 & 1 & 0 & 4 & 4 & 0 & 2 & 4 & 1 & 2 & 2 & 0 & 3 & 3 \\
\hline QJY78233.1 & 2 & 2 & 3 & 1 & 1 & 1 & 0 & 1 & 0 & 3 & 3 & 0 & 2 & 3 & 1 & 2 & 1 & 0 & 3 & 4 \\
\hline QVL90897.1 & 2 & 2 & 4 & 2 & 1 & 1 & 0 & 1 & 0 & 3 & 4 & 0 & 2 & 4 & 1 & 2 & 2 & 0 & 3 & 4 \\
\hline QOH29638.1 & 2 & 2 & 5 & 1 & 2 & 1 & 0 & 1 & 0 & 3 & 4 & 0 & 2 & 3 & 1 & 2 & 2 & 0 & 3 & 4 \\
\hline QWC74916.1 & 2 & 2 & 4 & 1 & 1 & 1 & 0 & 1 & 0 & 3 & 4 & 0 & 2 & 4 & 1 & 2 & 2 & 0 & 3 & 4 \\
\hline QSL79091.1 & 2 & 2 & 4 & 1 & 1 & 1 & 0 & 1 & 0 & 3 & 4 & 1 & 2 & 4 & 1 & 2 & 2 & 0 & 3 & 4 \\
\hline BCX23983.1 & 2 & 2 & 5 & 1 & 1 & 1 & 0 & 1 & 0 & 3 & 3 & 0 & 2 & 4 & 1 & 2 & 1 & 0 & 3 & 4 \\
\hline QWY94400.1 & 2 & 2 & 5 & 1 & 1 & 1 & 0 & 1 & 0 & 3 & 3 & 0 & 2 & 5 & 1 & 2 & 2 & 0 & 3 & 4 \\
\hline QSE25736.1 & 2 & 2 & 5 & 1 & 1 & 1 & 0 & 1 & 1 & 3 & 3 & 0 & 2 & 4 & 1 & 2 & 2 & 0 & 3 & 4 \\
\hline QWN58574.1 & 2 & 2 & 5 & 1 & 1 & 1 & 0 & 1 & 0 & 4 & 3 & 0 & 2 & 4 & 1 & 2 & 2 & 0 & 3 & 4 \\
\hline
\end{tabular}


Table 6: Frequency distribution of amino acids over the 140 unique SARS-CoV-2 ORF10 variants (from QTI75440.1 to QWY55420.1)

\begin{tabular}{|c|c|c|c|c|c|c|c|c|c|c|c|c|c|c|c|c|c|c|c|c|}
\hline ORF10 & A & $\mathrm{R}$ & $\mathrm{N}$ & $\mathrm{D}$ & $\mathrm{C}$ & Q & $\mathrm{E}$ & G & $\mathrm{H}$ & I & $\mathrm{L}$ & K & M & $\mathrm{F}$ & $\mathrm{P}$ & $\mathrm{S}$ & $\mathrm{T}$ & W & Y & $\mathrm{V}$ \\
\hline QTI75440.1 & 3 & 2 & 5 & 1 & 1 & 1 & 0 & 1 & 0 & 3 & 4 & 0 & 2 & 4 & 1 & 2 & 1 & 0 & 3 & 4 \\
\hline QWS53172.1 & 2 & 2 & 5 & 1 & 1 & 1 & 0 & 1 & 0 & 4 & 4 & 0 & 2 & 4 & 1 & 2 & 1 & 0 & 3 & 4 \\
\hline YP_009725255.1 & 2 & 2 & 5 & 1 & 1 & 1 & 0 & 1 & 0 & 3 & 4 & 0 & 2 & 4 & 1 & 2 & 2 & 0 & 3 & 4 \\
\hline QWY95092.1 & 2 & 2 & 5 & 1 & 1 & 1 & 0 & 1 & 0 & 3 & 3 & 0 & 2 & 4 & 2 & 2 & 2 & 0 & 3 & 4 \\
\hline QWQ66744.1 & 2 & 3 & 5 & 1 & 1 & 1 & 0 & 1 & 0 & 3 & 3 & 0 & 2 & 4 & 1 & 2 & 2 & 0 & 3 & 4 \\
\hline QSW62483.1 & 2 & 2 & 4 & 1 & 1 & 1 & 0 & 1 & 0 & 3 & 4 & 0 & 2 & 4 & 1 & 3 & 2 & 0 & 3 & 4 \\
\hline QVU28280.1 & 2 & 2 & 5 & 1 & 1 & 1 & 0 & 1 & 0 & 3 & 5 & 0 & 2 & 3 & 1 & 2 & 2 & 0 & 3 & 4 \\
\hline QWW53635.1 & 2 & 2 & 5 & 1 & 1 & 1 & 0 & 1 & 0 & 3 & 4 & 0 & 2 & 3 & 1 & 3 & 2 & 0 & 3 & 4 \\
\hline QQX05795.1 & 2 & 2 & 4 & 1 & 1 & 1 & 0 & 1 & 0 & 3 & 4 & 0 & 2 & 4 & 1 & 2 & 2 & 0 & 4 & 4 \\
\hline QVE28736.1 & 2 & 2 & 5 & 0 & 1 & 1 & 0 & 2 & 0 & 3 & 4 & 0 & 2 & 4 & 1 & 2 & 2 & 0 & 3 & 4 \\
\hline QTC84700.1 & 2 & 2 & 5 & 0 & 1 & 1 & 0 & 1 & 1 & 3 & 4 & 0 & 2 & 4 & 1 & 2 & 2 & 0 & 3 & 4 \\
\hline QWN49673.1 & 2 & 2 & 6 & 1 & 1 & 1 & 0 & 1 & 0 & 3 & 4 & 0 & 2 & 4 & 1 & 2 & 2 & 0 & 3 & 3 \\
\hline QUA36764.1 & 2 & 2 & 6 & 0 & 1 & 1 & 0 & 1 & 0 & 3 & 4 & 0 & 2 & 4 & 1 & 2 & 2 & 0 & 3 & 4 \\
\hline QSSW42096.1 & 2 & 2 & 5 & 0 & 1 & 1 & 0 & 1 & 0 & 3 & 4 & 0 & 2 & 4 & 1 & 2 & 2 & 0 & 3 & 5 \\
\hline QWY22460.1 & 2 & 2 & 5 & 0 & 1 & 1 & 0 & 1 & 0 & 3 & 4 & 0 & 2 & 4 & 1 & 2 & 2 & 0 & 4 & 4 \\
\hline QUF19963.1 & 2 & 3 & 5 & 1 & 1 & 0 & 0 & 1 & 0 & 3 & 4 & 0 & 2 & 4 & 1 & 2 & 2 & 0 & 3 & 4 \\
\hline QTB11041.1 & 1 & 2 & 5 & 1 & 1 & 1 & 0 & 1 & 0 & 3 & 4 & 0 & 2 & 4 & 2 & 2 & 2 & 0 & 3 & 4 \\
\hline QWX09518.1 & 1 & 2 & 5 & 1 & 1 & 1 & 0 & 1 & 0 & 3 & 4 & 0 & 2 & 4 & 1 & 3 & 2 & 0 & 3 & 4 \\
\hline QWU01215.1 & 1 & 2 & 5 & 1 & 1 & 1 & 0 & 1 & 0 & 3 & 4 & 0 & 2 & 4 & 1 & 2 & 2 & 0 & 3 & 5 \\
\hline QRM91569.1 & 2 & 2 & 5 & 1 & 1 & 1 & 0 & 1 & 0 & 2 & 4 & 1 & 2 & 4 & 1 & 2 & 2 & 0 & 3 & 4 \\
\hline QRX03618.1 & 2 & 2 & 5 & 1 & 1 & 1 & 0 & 1 & 0 & 2 & 5 & 0 & 2 & 4 & 1 & 2 & 2 & 0 & 3 & 4 \\
\hline QSO40790.1 & 2 & 2 & 5 & 1 & 1 & 1 & 0 & 1 & 0 & 2 & 4 & 0 & 3 & 4 & 1 & 2 & 2 & 0 & 3 & 4 \\
\hline QVE25858.1 & 2 & 3 & 5 & 1 & 1 & 1 & 0 & 1 & 0 & 2 & 4 & 0 & 2 & 4 & 1 & 2 & 2 & 0 & 3 & 4 \\
\hline QVJ17584.1 & 2 & 2 & 5 & 1 & 1 & 1 & 0 & 1 & 0 & 2 & 4 & 0 & 2 & 4 & 1 & 2 & 3 & 0 & 3 & 4 \\
\hline QVO95200.1 & 2 & 2 & 5 & 1 & 1 & 1 & 0 & 1 & 0 & 2 & 4 & 0 & 2 & 4 & 1 & 2 & 2 & 0 & 3 & 5 \\
\hline QVE30392.1 & 2 & 2 & 4 & 1 & 1 & 1 & 0 & 1 & 0 & 3 & 4 & 0 & 2 & 4 & 1 & 3 & 2 & 0 & 3 & 4 \\
\hline QTC19517.1 & 2 & 1 & 5 & 1 & 1 & 1 & 0 & 1 & 0 & 3 & 4 & 0 & 2 & 4 & 1 & 3 & 2 & 0 & 3 & 4 \\
\hline QVJ37366.1 & 2 & 2 & 4 & 1 & 1 & 1 & 0 & 1 & 0 & 3 & 4 & 0 & 2 & 4 & 1 & 3 & 2 & 0 & 3 & 4 \\
\hline QWQ76823.1 & 2 & 2 & 4 & 1 & 1 & 1 & 0 & 1 & 0 & 3 & 4 & 0 & 2 & 4 & 1 & 2 & 3 & 0 & 3 & 4 \\
\hline QVV08801.1 & 2 & 2 & 4 & 1 & 1 & 1 & 0 & 1 & 0 & 3 & 4 & 0 & 2 & 4 & 1 & 2 & 2 & 0 & 4 & 4 \\
\hline QUI12106.1 & 2 & 2 & 5 & 1 & 1 & 1 & 0 & 1 & 0 & 3 & 4 & 0 & 1 & 4 & 1 & 2 & 3 & 0 & 3 & 4 \\
\hline QWU53472.1 & 2 & 2 & 5 & 1 & 1 & 1 & 0 & 1 & 0 & 3 & 4 & 0 & 1 & 4 & 1 & 2 & 2 & 0 & 3 & 5 \\
\hline QTS24551.1 & 2 & 1 & 5 & 1 & 1 & 1 & 0 & 1 & 0 & 3 & 4 & 0 & 2 & 4 & 1 & 2 & 3 & 0 & 3 & 4 \\
\hline QNV50343.1 & 2 & 2 & 5 & 1 & 0 & 1 & 0 & 1 & 0 & 3 & 4 & 0 & 2 & 5 & 1 & 2 & 2 & 0 & 3 & 4 \\
\hline QUB17908.1 & 1 & 2 & 5 & 1 & 0 & 1 & 0 & 1 & 0 & 3 & 4 & 0 & 2 & 5 & 1 & 2 & 2 & 0 & 3 & 5 \\
\hline QVL64016.1 & 2 & 2 & 5 & 1 & 1 & 1 & 0 & 1 & 0 & 3 & 3 & 0 & 2 & 4 & 2 & 2 & 2 & 0 & 3 & 4 \\
\hline QWU68360.1 & 2 & 2 & 5 & 1 & 1 & 1 & 0 & 1 & 0 & 3 & 3 & 0 & 2 & 4 & 2 & 2 & 2 & 0 & 3 & 4 \\
\hline QTO29824.1 & 2 & 2 & 5 & 1 & 1 & 1 & 0 & 1 & 0 & 2 & 5 & 0 & 2 & 4 & 1 & 2 & 2 & 0 & 3 & 4 \\
\hline QWU51246.1 & 2 & 2 & 5 & 1 & 1 & 1 & 0 & 1 & 0 & 2 & 4 & 0 & 3 & 4 & 1 & 2 & 2 & 0 & 3 & 4 \\
\hline QVX69392.1 & 2 & 2 & 5 & 1 & 1 & 1 & 0 & 1 & 0 & 2 & 4 & 0 & 2 & 4 & 1 & 2 & 3 & 0 & 3 & 4 \\
\hline QWO21857.1 & 2 & 2 & 5 & 1 & 1 & 1 & 0 & 1 & 0 & 2 & 4 & 0 & 2 & 4 & 1 & 2 & 2 & 0 & 3 & 5 \\
\hline QTK02152.1 & 2 & 2 & 5 & 1 & 1 & 1 & 0 & 1 & 0 & 3 & 5 & 0 & 2 & 3 & 1 & 2 & 2 & 0 & 3 & 4 \\
\hline QWY72735.1 & 2 & 2 & 5 & 1 & 1 & 1 & 0 & 1 & 0 & 3 & 4 & 0 & 2 & 3 & 1 & 3 & 2 & 0 & 3 & 4 \\
\hline QVH90751.1 & 2 & 2 & 5 & 1 & 1 & 2 & 0 & 1 & 0 & 3 & 4 & 0 & 2 & 4 & 0 & 2 & 2 & 0 & 3 & 4 \\
\hline QVX36355.1 & 2 & 1 & 5 & 1 & 1 & 1 & 0 & 1 & 0 & 3 & 5 & 0 & 2 & 4 & 0 & 3 & 2 & 0 & 3 & 4 \\
\hline QWY95104.1 & 2 & 2 & 5 & 1 & 1 & 1 & 0 & 1 & 0 & 3 & 3 & 0 & 2 & 5 & 0 & 3 & 2 & 0 & 3 & 4 \\
\hline QWWY18545.1 & 2 & 2 & 5 & 1 & 1 & 1 & 0 & 1 & 0 & 3 & 4 & 0 & 2 & 4 & 0 & 3 & 2 & 0 & 3 & 4 \\
\hline QVL15727.1 & 2 & 2 & 5 & 1 & 1 & 1 & 0 & 1 & 0 & 3 & 4 & 0 & 2 & 4 & 0 & 2 & 3 & 0 & 3 & 4 \\
\hline QTO07027.1 & 2 & 2 & 5 & 1 & 1 & 1 & 0 & 1 & 0 & 3 & 4 & 0 & 2 & 3 & 0 & 2 & 2 & 0 & 3 & 4 \\
\hline BCX25240.1 & 2 & 2 & 5 & 1 & 1 & 1 & 0 & 1 & 0 & 3 & 5 & 0 & 2 & 3 & 1 & 2 & 2 & 0 & 3 & 4 \\
\hline QWB65585.1 & 2 & 2 & 5 & 1 & 1 & 1 & 0 & 1 & 0 & 3 & 4 & 0 & 2 & 3 & 1 & 3 & 2 & 0 & 3 & 4 \\
\hline QTW57386.1 & 2 & 2 & 5 & 1 & 1 & 1 & 0 & 1 & 0 & 3 & 4 & 0 & 2 & 3 & 1 & 2 & 2 & 0 & 4 & 4 \\
\hline QWW38212.1 & 1 & 2 & 5 & 2 & 1 & 1 & 0 & 1 & 0 & 3 & 4 & 0 & 2 & 4 & 1 & 2 & 2 & 0 & 3 & 4 \\
\hline QSE30156.1 & 1 & 2 & 5 & 1 & 1 & 1 & 0 & 2 & 0 & 3 & 4 & 0 & 2 & 4 & 1 & 2 & 2 & 0 & 3 & 4 \\
\hline QUF17645.1 & 1 & 2 & 5 & 1 & 1 & 1 & 0 & 1 & 0 & 3 & 4 & 0 & 2 & 4 & 2 & 2 & 2 & 0 & 3 & 4 \\
\hline QUF20717.1 & 1 & 2 & 5 & 1 & 1 & 1 & 0 & 1 & 0 & 3 & 4 & 0 & 2 & 3 & 1 & 2 & 2 & 0 & 3 & 4 \\
\hline QWB83606.1 & 1 & 2 & 5 & 1 & 1 & 1 & 0 & 1 & 0 & 3 & 4 & 0 & 2 & 4 & 1 & 3 & 2 & 0 & 3 & 4 \\
\hline QPF60767.1 & 1 & 2 & 5 & 1 & 1 & 1 & 0 & 1 & 0 & 3 & 4 & 0 & 2 & 4 & 1 & 2 & 3 & 0 & 3 & 4 \\
\hline QVR42407.1 & 1 & 2 & 5 & 1 & 1 & 1 & 0 & 1 & 0 & 3 & 4 & 0 & 2 & 2 & 0 & 2 & 2 & 0 & 3 & 4 \\
\hline QUA32182.1 & 1 & 2 & 5 & 1 & 1 & 1 & 0 & 1 & 0 & 3 & 5 & 0 & 2 & 4 & 0 & 2 & 2 & 0 & 3 & 5 \\
\hline QRG41735.1 & 1 & 1 & 5 & 1 & 1 & 1 & 0 & 1 & 0 & 3 & 5 & 0 & 2 & 4 & 1 & 2 & 2 & 0 & 3 & 5 \\
\hline QWT73590.1 & 1 & 2 & 5 & 1 & 1 & 1 & 0 & 1 & 0 & 3 & 4 & 0 & 2 & 4 & 1 & 2 & 2 & 0 & 3 & 5 \\
\hline QWK62266.1 & 2 & 2 & 5 & 1 & 1 & 1 & 0 & 1 & 0 & 3 & 5 & 0 & 2 & 3 & 1 & 2 & 2 & 0 & 3 & 4 \\
\hline QVM67662.1 & 2 & 2 & 5 & 1 & 1 & 1 & 0 & 1 & 0 & 4 & 4 & 0 & 2 & 3 & 1 & 3 & 1 & 0 & 3 & 4 \\
\hline QQE14148.1 & 2 & 2 & 5 & 1 & 1 & 1 & 0 & 1 & 0 & 3 & 4 & 0 & 2 & 3 & 1 & 3 & 2 & 0 & 3 & 4 \\
\hline QैVU00728.1 & 2 & 2 & 4 & 1 & 1 & 1 & 0 & 1 & 0 & 3 & 4 & 0 & 2 & 4 & 1 & 3 & 2 & 0 & 3 & 4 \\
\hline QUA79573.1 & 2 & 2 & 4 & 1 & 1 & 1 & 0 & 1 & 0 & 3 & 4 & 0 & 2 & 4 & 1 & 2 & 2 & 0 & 4 & 4 \\
\hline QWT65692.1 & 2 & 2 & 5 & 1 & 1 & 1 & 0 & 1 & 0 & 2 & 5 & 0 & 2 & 4 & 1 & 2 & 2 & 0 & 3 & 4 \\
\hline QVW43945.1 & 2 & 2 & 5 & 1 & 1 & 1 & 0 & 1 & 0 & 2 & 4 & 0 & 2 & 4 & 1 & 2 & 3 & 0 & 3 & 4 \\
\hline QUM21266.1 & 2 & 2 & 4 & 2 & 1 & 1 & 0 & 1 & 0 & 2 & 4 & 0 & 2 & 4 & 1 & 2 & 2 & 0 & 3 & 5 \\
\hline QTX06599.1 & 2 & 1 & 5 & 1 & 2 & 1 & 0 & 1 & 0 & 2 & 4 & 0 & 2 & 4 & 1 & 2 & 2 & 0 & 3 & 5 \\
\hline QVM14069.1 & 2 & 2 & 5 & 1 & 1 & 1 & 0 & 1 & 0 & 2 & 3 & 0 & 2 & 5 & 1 & 2 & 2 & 0 & 3 & 5 \\
\hline QWY55420.1 & 2 & 2 & 5 & 1 & 1 & 1 & 0 & 1 & 0 & 2 & 4 & 0 & 2 & 4 & 1 & 2 & 2 & 0 & 3 & 5 \\
\hline
\end{tabular}

It was noticed that asparagine was present in the ORF10 sequences with the highest frequencies ranging from 4 to 6 (Figure $6(\mathrm{~A})$ ). Also, the frequencies of L, F, and $\mathrm{V}$ in each 
ORF10 variant were dominant as compared to that of other amino acids.

For each pair of frequency vectors associated with ORF10 sequences, distances were enumerated (Figure 6(B)).
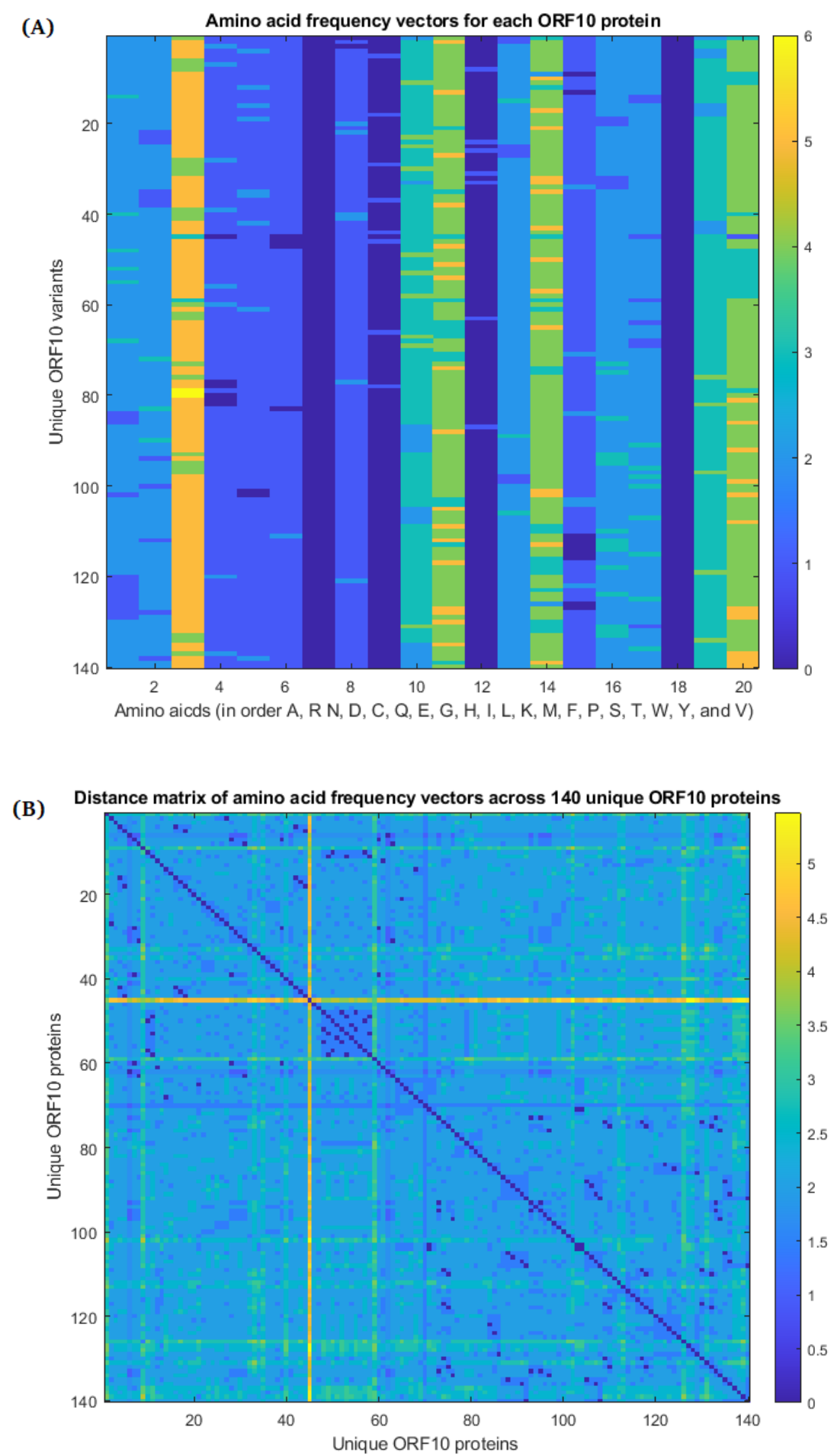

Figure 6. (A): Frequency distribution of amino acid across the unique 140 ORF10 proteins, and (B) Pairwise distance matrix of frequency vectors for each ORF10 protein 
The most distant frequency vector was detected for the SARS-CoV-2 ORF10 BCY15724.1 (Japan, collected in November, 2020). Based on frequency-vector similarity (Euclidean distance-wise nearness), unique 140 ORF10 proteins were clustered using the K-means clustering method (Table 7).

Table 7: K-means clustering (clusters: 20) of the unique ORF10 variants based on amino acid frequency distribution

\begin{tabular}{|c|c|c|c|c|c|c|c|}
\hline ORF10 & Cluster & ORF10 & Cluster & ORF10 & Cluster & ORF10 & Cluster \\
\hline QKM75696.1 & 8 & QVW78101.1 & 11 & BCY15724.1 & 17 & QWN58574.1 & 15 \\
\hline QKG88643.1 & 1 & QLA48060.1 & 1 & QWT72678.1 & 16 & QTI75440.1 & 16 \\
\hline QVJ13930.1 & 1 & QVO98764.1 & 11 & QUM42028.1 & 1 & QWS53172.1 & 11 \\
\hline QWE67724.1 & 7 & QWY66101.1 & 1 & QUX49292.1 & 14 & YP_009725255.1 & 5 \\
\hline QRJ36840.1 & 7 & QWQ05246.1 & 1 & QWK69365.1 & 11 & QWY95092.1 & 15 \\
\hline QQY03084.1 & 5 & QTS35265.1 & 20 & QTA74333.1 & 3 & QWQ66744.1 & 15 \\
\hline QRG22086.1 & 20 & QVP24786.1 & 20 & QWY54619.1 & 14 & QSW62483.1 & 5 \\
\hline QTD22916.1 & 20 & QVO85840.1 & 20 & QUV26065.1 & 14 & QVU28280.1 & 6 \\
\hline QUL69971.1 & 18 & QVO40425.1 & 20 & QWF05003.1 & 11 & QWW53635.1 & 19 \\
\hline QWB85197.1 & 3 & QWU52456.1 & 3 & QUP00476.1 & 14 & QQX05795.1 & 20 \\
\hline QWJ83116.1 & 11 & QTP28305.1 & 10 & QTJ93574.1 & 14 & QVE28736.1 & 16 \\
\hline QSE09446.1 & 6 & QWS07290.1 & 16 & QWN49685.1 & 14 & QTC84700.1 & 16 \\
\hline QWZ00470.1 & 1 & QWF07009.1 & 10 & QVO91006.1 & 3 & QWN49673.1 & 14 \\
\hline QTP26076.1 & 16 & QWY19801.1 & 1 & QVV18442.1 & 11 & QUA36764.1 & 16 \\
\hline QWK62875.1 & 16 & QWX30181.1 & 1 & QJY78233.1 & 8 & QSW42096.1 & 4 \\
\hline QVG57396.1 & 7 & QWY95666.1 & 1 & QVL90897.1 & 20 & QWY22460.1 & 16 \\
\hline QRA60944.1 & 3 & QVU00656.1 & 20 & QOH29638.1 & 6 & QUF19963.1 & 16 \\
\hline QWE68295.1 & 7 & QTA53643.1 & 16 & QWC74916.1 & 5 & QTB11041.1 & 4 \\
\hline QWT58729.1 & 16 & QUG14309.1 & 20 & QSL79091.1 & 20 & QWX09518.1 & 4 \\
\hline QWM42669.1 & 16 & QWY70751.1 & 7 & BCX23983.1 & 15 & QWU01215.1 & 4 \\
\hline QWS64226.1 & 3 & QSJ35636.1 & 3 & QWY94400.1 & 3 & QRM91569.1 & 9 \\
\hline QVJ47956.1 & 1 & QWF03959.1 & 7 & QSE25736.1 & 15 & QRX03618.1 & 12 \\
\hline ORF10 & Cluster & ORF10 & Cluster & ORF10 & Cluster & ORF10 & Cluster \\
\hline QSO40790.1 & 9 & QVH90751.1 & 1 & QVU00728.1 & 5 & QWU68360.1 & 15 \\
\hline QVE25858.1 & 9 & QVX36355.1 & 2 & QUA79573.1 & 20 & QTO29824.1 & 12 \\
\hline QVJ17584.1 & 9 & QWY95104.1 & 10 & QWT65692.1 & 12 & QWU51246.1 & 9 \\
\hline QVO95200.1 & 9 & QWY18545.1 & 1 & QVW43945.1 & 9 & QVX69392.1 & 9 \\
\hline QVE30392.1 & 5 & QVL15727.1 & 1 & QUM21266.1 & 10 & QWO21857.1 & 9 \\
\hline QTC19517.1 & 1 & QTO07027.1 & 6 & QTX06599.1 & 10 & QTK02152.1 & 6 \\
\hline QVJ37366.1 & 5 & BCX25240.1 & 6 & QVM14069.1 & 10 & QWY72735.1 & 19 \\
\hline QWQ76823.1 & 20 & QWB65585.1 & 19 & QWY55420.1 & 9 & & \\
\hline QVV08801.1 & 20 & QTW57386.1 & 6 & QVR42407.1 & 18 & & \\
\hline QUI12106.1 & 1 & QWW38212.1 & 4 & QUA32182.1 & 2 & & \\
\hline QWU53472.1 & 4 & QSE30156.1 & 4 & QRG41735.1 & 2 & & \\
\hline QTS24551.1 & 1 & QUU17645.1 & 4 & QWT73590.1 & 4 & & \\
\hline QNV50343.1 & 3 & QUF20717.1 & 6 & QWK62266.1 & 6 & & \\
\hline QUB17908.1 & 2 & QWB83606.1 & 4 & QVM67662.1 & 13 & & \\
\hline QVL64016.1 & 15 & QPF60767.1 & 4 & QQE14148.1 & 19 & & \\
\hline
\end{tabular}

Based on amino acid compositions in each ORF10 sequence, unique ORF10 proteins were clustered into 20 different clusters. Cluster- 1 contains the highest number (17) of ORF10 variants, which fall in the US, whereas clusters 13 and 17 contain only one ORF10 variant each. The ORF10 proteins BCY15724 (collected from Japan) and QVM67662 (USA:Florida) belong to cluster 13 and 17, respectively (Table 8). 
Table 8: Frequency of ORF10 variants in each cluster

\begin{tabular}{cccccc}
\hline Cluster Number & Frequency & Cluster Number & Frequency & Cluster Number & Frequency \\
\hline 1 & 17 & 5 & 7 & 19 & 4 \\
20 & 14 & 11 & 7 & 12 & 3 \\
16 & 13 & 14 & 7 & 8 & 2 \\
4 & 11 & 15 & 7 & 18 & 2 \\
9 & 10 & 7 & 6 & 13 & 1 \\
3 & 9 & 10 & 6 & 17 & 1 \\
6 & 9 & 2 & 4 & & \\
\hline
\end{tabular}

Clearly, twenty different clusters with different frequencies of ORF10 variants showed wide variations of ORF10 sequences based on amino acid compositions.

\section{Discussion}

In this study, a total of 140 unique SARS-CoV-2 ORF10 sequences were observed among 202968 ORF10 sequences obtained from the NCBI database. Remarkably, most unique ORF10 variants were first reported in North America only (Table 1). Consequently, the highest number of point mutations and co-mutation were found in North America. Continent-wise Africa had the highest percentage (1.27\%) of unique variants of ORF10 sequences from Africa. Furthermore, it was observed that all amino acids of ORF10 except the residue 18, possessed single-missense mutations (Table 2). The unique ORF10 variants from Asia, Africa, and Oceania had several point-mutations at common residue positions (Table 2). We noticed that in North America, ORF10 mutations only at the amino acid residue positions 11 (F11S, F11L) and 16 (L16P) were deleterious, as reported from North America. A significant percentage of non-synonymous mutations, 53 among the total of 128 mutations were deleterious, which may alter the intensity of the interactome between ORF10 and host proteins. The 22 unique co-mutations were observed in different geo-locations Table 4. It was noticed that co-mutations in ORF10 variants became an emerging trend, which was varies slightly in 2020, and inevitably in the future ORF10 variants with co-mutations might be transmitted to other geo-locations while restriction on international travel is lifted. Furthermore, it was observed that the effects of co-occurring mutants are qualitatively similar to those of single mutations, as most variability is at the disorder predisposition of $\mathrm{N}$ - and $\mathrm{C}$-terminal regions and a region centered at residue 25 . However, in co-occurring mutants, scales of changes at the terminal regions are noticeably larger. Most of the co-occurring mutations increase the disorder predisposition of N-terminal region of ORF10, whereas many such mutations decrease the disorder propensity of the region centered at residue 25. Consequently, these mutations may affect secondary structure and associated functions of the ORF10 variants. In addition, amino acid residue positions of the secondary structures (especially the N-terminal $\alpha$-helix regions) of several ORF10 variants changed, and therefore interaction of those ORF10 variants with the human protein $C U L 2^{Z Y G 11 B}$ complex are most likely affected.

\section{Conclusions}

Unique variations in SARS-CoV-2 ORF10 proteins are an emerging trend across different continents due to the appearance of various single point and co-occurring mutations. The highest percentage of unique ORF10 variants were found in Africa, though the frequency of unique ORF10 variants was significantly dominating with a total number of unique variants available. It was further observed that the growth rate of emerging non-synonymous mutations in ORF10 proteins is increasing nonlinearly, which is certainly alarming with regards to stability or instability of emerging SARS-CoV-2 variants. Due to significant deleterious mutations, expression of the SARSCoV-2 ORF10 proteins might get altered a phenomenon, which will affect functional virus-host protein-protein interactions. Also, it was reported that secondary structure 
$\alpha$-helix regions of the ORF10 variants was changed due to co-mutations. Consequently, virulence/pathogenicity of SARS-CoV-2 may get influenced directly or indirectly and therefore, continous surveillance of mutations and their associated effects is necessary.

Author Contributions: SSH and VU designed research and performed research. SSH, VU wrote the primary draft of the article. KL, ASA, PA, AAAA, AL, RK, TMAEA, PPC, GKA, SS, MT, GC, $\mathrm{KT}, \mathrm{DB}$, and GP edited the manuscript. EMR and $\mathrm{PB}$ proofread the final article. All authors read the final article, and approved for submission.

Funding: This research received no external funding.

Acknowledgments: We are grateful to Agnel Praveen Joseph of the Science and Technology Facilities Council, UKRI, UK for his kind help in getting several information related to the webserver CoVal.

Conflicts of Interest: Authors declare no competing interest to declare.

\section{References}

1. Worldometer. COVID Live Update. https:/ /www.worldometers.info/coronavirus/, 2010 (accessed July 16, 2021).

2. Johns-Hopkin-University. Coronavirus Resource Center. https://coronavirus.jhu.edu/map.html, 2010 (accessed July 16, 2021).

3. Laha, S.; Chakraborty, J.; Das, S.; Manna, S.K.; Biswas, S.; Chatterjee, R. Characterizations of SARS-CoV-2 mutational profile, spike protein stability and viral transmission. Infection, Genetics and Evolution 2020, 85, 104445.

4. Kim, J.S.; Jang, J.H.; Kim, J.M.; Chung, Y.S.; Yoo, C.K.; Han, M.G. Genome-wide identification and characterization of point mutations in the SARS-CoV-2 genome. Osong Public Health and Research Perspectives 2020, 11, 101.

5. Weber, S.; Ramirez, C.M.; Weiser, B.; Burger, H.; Doerfler, W. SARS-CoV-2 worldwide replication drives rapid rise and selection of mutations across the viral genome: a time-course study-potential challenge for vaccines and therapies. EMBO Molecular Medicine 2021, p. e14062.

6. Krause, P.R.; Fleming, T.R.; Longini, I.M.; Peto, R.; Briand, S.; Heymann, D.L.; Beral, V.; Snape, M.D.; Rees, H.; Ropero, A.M.; others. SARS-CoV-2 Variants and Vaccines. New England Journal of Medicine 2021.

7. Touret, F.; Luciani, L.; Baronti, C.; Cochin, M.; Driouich, J.S.; Gilles, M.; Thirion, L.; Nougairède, A.; de Lamballerie, X. Replicative Fitness of a SARS-CoV-2 20I/501Y. V1 Variant from Lineage B. 1.1. 7 in Human Reconstituted Bronchial Epithelium. Mbio 2021, 12, e00850-21.

8. Maurano, M.T.; Ramaswami, S.; Zappile, P.; Dimartino, D.; Boytard, L.; Ribeiro-dos Santos, A.M.; Vulpescu, N.A.; Westby, G.; Shen, G.; Feng, X.; others. Sequencing identifies multiple early introductions of SARS-CoV-2 to the New York City region. Genome research 2020, 30, 1781-1788.

9. Bi, C.; Ramos-Mandujano, G.; Tian, Y.; Hala, S.; Xu, J.; Mfarrej, S.; Esteban, C.R.; Delicado, E.N.; Alofi, F.S.; Khogeer, A.; others. Simultaneous detection and mutation surveillance of SARS-CoV-2 and multiple respiratory viruses by rapid field-deployable sequencing. Med 2021.

10. Satarker, S.; Nampoothiri, M. Structural proteins in severe acute respiratory syndrome coronavirus-2. Archives of medical research 2020, 51, 482-491.

11. Wang, D.; Jiang, A.; Feng, J.; Li, G.; Guo, D.; Sajid, M.; Wu, K.; Zhang, Q.; Ponty, Y.; Will, S.; others. The SARS-CoV-2 subgenome landscape and its novel regulatory features. Molecular cell 2021, 81, 2135-2147.

12. Kasibhatla, S.M.; Kinikar, M.; Limaye, S.; Kale, M.M.; Kulkarni-Kale, U. Understanding evolution of SARS-CoV-2: a perspective from analysis of genetic diversity of RdRp gene. Journal of medical virology 2020, 92, 1932-1937.

13. Michel, C.J.; Mayer, C.; Poch, O.; Thompson, J.D. Characterization of accessory genes in coronavirus genomes. Virology Journal 2020, 17, 1-13.

14. Silva, S.J.R.d.; Silva, C.T.A.d.; Mendes, R.P.G.; Pena, L.; others. Role of nonstructural proteins in the pathogenesis of SARS-CoV-2 2020.

15. Hillen, H.S.; Kokic, G.; Farnung, L.; Dienemann, C.; Tegunov, D.; Cramer, P. Structure of replicating SARS-CoV-2 polymerase. Nature 2020, 584, 154-156.

16. Clark, L.K.; Green, T.J.; Petit, C.M. Structure of nonstructural protein 1 from SARS-CoV-2. Journal of Virology 2021, 95, e02019-20.

17. Shannon, A.; Le, N.T.T.; Selisko, B.; Eydoux, C.; Alvarez, K.; Guillemot, J.C.; Decroly, E.; Peersen, O.; Ferron, F.; Canard, B. Remdesivir and SARS-CoV-2: Structural requirements at both nsp12 RdRp and nsp14 Exonuclease active-sites. Antiviral research 2020, 178, 104793.

18. Duffy, S. Why are RNA virus mutation rates so damn high? PLoS biology 2018, 16, e3000003.

19. Elena, S.F.; Sanjuán, R. Adaptive value of high mutation rates of RNA viruses: separating causes from consequences. Journal of virology 2005, 79, 11555-11558.

20. Yan, L.; Yang, Y.; Li, M.; Zhang, Y.; Zheng, L.; Ge, J.; Huang, Y.C.; Liu, Z.; Wang, T.; Gao, S.; others. Coupling of N7methyltransferase and 3/-5/ exoribonuclease with SARS-CoV-2 polymerase reveals mechanisms for capping and proofreading. Cell 2021, 184, 3474-3485. 
21. Chen, J.; Wang, R.; Wang, M.; Wei, G.W. Mutations strengthened SARS-CoV-2 infectivity. Journal of molecular biology 2020, 432, 5212-5226.

22. Eskier, D.; Karakülah, G.; Suner, A.; Oktay, Y. RdRp mutations are associated with SARS-CoV-2 genome evolution. PeerJ 2020, 8, e9587.

23. Gupta, A.M.; Chakrabarti, J.; Mandal, S. Non-synonymous mutations of SARS-CoV-2 leads epitope loss and segregates its variants. Microbes and infection 2020, 22, 598-607.

24. Hassan, S.S.; Choudhury, P.P.; Roy, B. SARS-CoV2 envelope protein: non-synonymous mutations and its consequences. Genomics 2020, 112, 3890-3892.

25. Issa, E.; Merhi, G.; Panossian, B.; Salloum, T.; Tokajian, S. SARS-CoV-2 and ORF3a: nonsynonymous mutations, functional domains, and viral pathogenesis. Msystems 2020, 5, e00266-20.

26. Seyran, M.; Pizzol, D.; Adadi, P.; El-Aziz, T.M.; Hassan, S.S.; Soares, A.; Kandimalla, R.; Lundstrom, K.; Tambuwala, M.; Aljabali, A.A.; others. Questions concerning the proximal origin of SARS-CoV-2. Journal of Medical Virology 2021, 93, 1204.

27. Narayanan, K.; Huang, C.; Makino, S. SARS coronavirus accessory proteins. Virus research 2008, 133, $113-121$.

28. Hassan, S.S.; Attrish, D.; Ghosh, S.; Choudhury, P.P.; Uversky, V.N.; Aljabali, A.A.; Lundstrom, K.; Uhal, B.D.; Rezaei, N.; Seyran, M.; others. Notable sequence homology of the ORF10 protein introspects the architecture of SARS-COV-2. International Journal of Biological Macromolecules 2021, 181, 801-809.

29. Schuster, N.A. Characterization and structural prediction of the putative ORF10 protein in SARS-CoV-2. bioRxiv 2021, pp. 2020-10.

30. Pancer, K.; Milewska, A.; Owczarek, K.; Dabrowska, A.; Kowalski, M.; Łabaj, P.P.; Branicki, W.; Sanak, M.; Pyrc, K. The SARS-CoV-2 ORF10 is not essential in vitro or in vivo in humans. PLoS Pathogens 2020, 16, e1008959.

31. Altincekic, N.; Korn, S.M.; Qureshi, N.S.; Dujardin, M.; Ninot-Pedrosa, M.; Abele, R.; Abi Saad, M.J.; Alfano, C.; Almeida, F.C.; Alshamleh, I.; others. Large-scale recombinant production of the SARS-CoV-2 proteome for high-throughput and structural biology applications. Frontiers in molecular biosciences 2021, 8, 89.

32. Gordon, D.E.; Jang, G.M.; Bouhaddou, M.; Xu, J.; Obernier, K.; White, K.M.; O’Meara, M.J.; Rezelj, V.V.; Guo, J.Z.; Swaney, D.L.; others. A SARS-CoV-2 protein interaction map reveals targets for drug repurposing. Nature 2020, 583, 459-468.

33. Mena, E.L.; Donahue, C.J.; Vaites, L.P.; Li, J.; Rona, G.; O'Leary, C.; Lignitto, L.; Miwatani-Minter, B.; Paulo, J.A.; Dhabaria, A.; others. ORF10-Cullin-2-ZYG11B complex is not required for SARS-CoV-2 infection. Proceedings of the National Academy of Sciences 2021, 118.

34. Li, J.; Guo, M.; Tian, X.; Wang, X.; Yang, X.; Wu, P.; Liu, C.; Xiao, Z.; Qu, Y.; Yin, Y.; others. Virus-host interactome and proteomic survey reveal potential virulence factors influencing SARS-CoV-2 pathogenesis. Med 2021, 2, 99-112.

35. Yang, D.M.; Lin, F.C.; Tsai, P.H.; Chien, Y.; Wang, M.L.; Yang, Y.P.; Chang, T.J. Pandemic analysis of infection and death correlated with genomic open reading frame 10 mutation in severe acute respiratory syndrome coronavirus 2 victims. Journal of the Chinese Medical Association 2021, 84, 478-484.

36. Cagliani, R.; Forni, D.; Clerici, M.; Sironi, M. Coding potential and sequence conservation of SARS-CoV-2 and related animal viruses. Infection, Genetics and Evolution 2020, 83, 104353.

37. Käll, L.; Krogh, A.; Sonnhammer, E.L. A combined transmembrane topology and signal peptide prediction method. Journal of molecular biology 2004, 338, 1027-1036.

38. Madeira, F.; Park, Y.M.; Lee, J.; Buso, N.; Gur, T.; Madhusoodanan, N.; Basutkar, P.; Tivey, A.R.; Potter, S.C.; Finn, R.D.; others. The EMBL-EBI search and sequence analysis tools APIs in 2019. Nucleic acids research 2019, 47, W636-W641.

39. Drozdetskiy, A.; Cole, C.; Procter, J.; Barton, G.J. JPred4: a protein secondary structure prediction server. Nucleic acids research 2015, 43, W389-W394.

40. Obradovic, Z.; Peng, K.; Vucetic, S.; Radivojac, P.; Dunker, A.K. Exploiting heterogeneous sequence properties improves prediction of protein disorder. Proteins: Structure, Function, and Bioinformatics 2005, 61, 176-182.

41. Meng, F.; Uversky, V.N.; Kurgan, L. Comprehensive review of methods for prediction of intrinsic disorder and its molecular functions. Cellular and Molecular Life Sciences 2017, 74, 3069-3090.

42. Peng, Z.L.; Kurgan, L. Comprehensive comparative assessment of in-silico predictors of disordered regions. Current Protein and Peptide Science 2012, 13, 6-18.

43. Fan, X.; Kurgan, L. Accurate prediction of disorder in protein chains with a comprehensive and empirically designed consensus. Journal of Biomolecular Structure and Dynamics 2014, 32, 448-464.

44. Pickett, B.E.; Sadat, E.L.; Zhang, Y.; Noronha, J.M.; Squires, R.B.; Hunt, V.; Liu, M.; Kumar, S.; Zaremba, S.; Gu, Z.; others. ViPR: an open bioinformatics database and analysis resource for virology research. Nucleic acids research 2012, 40, D593-D598.

45. Bendl, J.; Stourac, J.; Salanda, O.; Pavelka, A.; Wieben, E.D.; Zendulka, J.; Brezovsky, J.; Damborsky, J. PredictSNP: robust and accurate consensus classifier for prediction of disease-related mutations. PLoS computational biology 2014, 10, e1003440.

46. Capriotti, E.; Fariselli, P. PhD-SNPg: a webserver and lightweight tool for scoring single nucleotide variants. Nucleic acids research 2017, 45, W247-W252.

47. Henson, R.; Cetto, L. The MATLAB bioinformatics toolbox. Encyclopedia of genetics, genomics, proteomics and bioinformatics 2004.

48. Hassan, S.S.; Aljabali, A.A.; Panda, P.K.; Ghosh, S.; Attrish, D.; Choudhury, P.P.; Seyran, M.; Pizzol, D.; Adadi, P.; Abd El-Aziz, T.M.; others. A unique view of SARS-CoV-2 through the lens of ORF8 protein. Computers in biology and medicine 2021, 133, 104380. 
49. Hassan, S.S.; Choudhury, P.P.; Roy, B.; Jana, S.S. Missense mutations in SARS-CoV2 genomes from Indian patients. Genomics 2020, 112, 4622-4627.

50. Mészáros, B.; Erdős, G.; Dosztányi, Z. IUPred2A: context-dependent prediction of protein disorder as a function of redox state and protein binding. Nucleic acids research 2018, 46, W329-W337. 